\title{
BKP and projective Hurwitz numbers
}

\author{
Sergei M. Natanzon* Aleksandr Yu. Orlov ${ }^{\dagger}$
}

November 29, 2016

\begin{abstract}
We consider $d$-fold branched coverings of the projective plane $\mathbb{R P}^{2}$ and show that the hypergeometric tau function of the BKP hierarchy of Kac and van de Leur is the generating function for weighted sums of the related Hurwitz numbers. In particular we get the $\mathbb{R} \mathbb{P}^{2}$ analogues of the $\mathbb{C P}^{1}$ generating functions proposed by Okounkov and by Goulden and Jackson. Other examples are Hurwitz numbers weighted by the Hall-Littlewood and by the Macdonald polynomials. We also consider integrals of tau functions which generate projective Hurwitz numbers and Hurwitz numbers related to different Euler characterisitics of the base Klein surfaces.
\end{abstract}

Key words: Hurwitz numbers, tau functions, BKP, projective plane, Schur polynomials, HallLittlewood polynomials, hypergeometric functions, random partitions, random matrices

2010 Mathematic Subject Classification: 05A15, 14N10, 17B80, 35Q51, 35Q53, 35Q55, 37K20, $37 \mathrm{~K} 30$,

\section{Introduction}

In 51] A. Okounkov studied ramified coverings of the Riemann sphere, having arbitrary ramification type over 0 and $\infty$, and with simple ramifications elsewhere, and made the seminal observation that the generating function for the related Hurwitz numbers (numbers of nonequivalent coverings with given ramification type) is a tau function for the Toda lattice hierarchy. Later links between the study of covers and integrable system were further developed by Okounkov and Pandharipande 52 and by Goulden and Jackson [19. Then a number of papers concerning the topic was written [44, [1], 14, [63, 23], 28, [29]. The review of this topic may be found in [33] and in [30. For the most recent works see [3] and references therein.

On the other hand it was shown that certain matrix models also generate Hurwitz numbers [63, [21, 35, 24, [10. It is not so surprising since tau functions used for generating of Hurwitz numbers belong to a special family found in [36] and in 55], [57] which are called tau functions of hypergeometric type, and such tau functions were used as asymptotic expansion of matrix integrals in [26, 60], [59, [27. Hypergeometric tau functions are multivariable generalizations of hypergeometric series where the role of Gauss hypergeometric equation plays the so-called string equation [55] and matrix integrals may be viewed as an analogue of the integral representation of Gauss hypergeometric series.

All works on Hurwitz numbers cited above are devoted to the counting of covers of the Riemann sphere and the links of this problem to the Toda lattice (TL) and Kadomtsev-Petviashvili (KP) hierarchies.

The covering problem of the Riemann sphere (Euler characteristic $\mathrm{E}=2$ ) goes back to classical results by Frobenius and Schur [16,17. We can refer the readers to the wonderful textbook [37 where the general case of the enumeration of covers of Riemann surfaces of higher genus was considered. The Frobenius-type formula for the Hurwitz numbers enumerating $d$-fold branched coverings of connected Riemann or Klein surfaces (without boundary) of any Euler characteristic E was obtained in the papers

\footnotetext{
*National Research University Higher School of Economics, Moscow, Russia; Institute for Theoretical and Experimental Physics, Moscow, Russia; email: natanzons@mail.ru

${ }^{\dagger}$ Institute of Oceanology, Nahimovskii Prospekt 36, Moscow 117997, Russia, and National Research University Higher School of Economics, International Laboratory of Representation Theory and Mathematical Physics, 20 Myasnitskaya Ulitsa, Moscow 101000, Russia, email: orlovs@ocean.ru
} 
of A. Mednykh and G. Pozdnyakova [42, 43] and also Gareth A. Jones [18. It contains the sum over irreducible representations $\lambda$ of the symmetric group $S_{d}$ (see [16, 18, 37, 42, 43])

$$
H^{\mathrm{E}, \mathrm{F}}\left(d ; \Delta^{(1)} \ldots, \Delta^{(\mathrm{F})}\right)=\sum_{\lambda}\left(\frac{\operatorname{dim} \lambda}{d !}\right)^{\mathrm{E}} \prod_{i=1}^{\mathrm{F}} \varphi_{\lambda}\left(\Delta^{(i)}\right),
$$

where $\mathrm{E}$ is the Euler characteristic of the base surface $\Omega, \Delta^{(i)}$ are profiles over branch points on $\Omega, \operatorname{dim} \lambda$ is the dimension of the irreducible representation of $S_{d}$, and

$$
\varphi_{\lambda}\left(\Delta^{(i)}\right):=\left|C_{\Delta^{(i)}}\right| \frac{\chi_{\lambda}\left(\Delta^{(i)}\right)}{\operatorname{dim} \lambda}, \quad \operatorname{dim} \lambda:=\chi_{\lambda}\left(\left(1^{d}\right)\right)
$$

$\chi_{\lambda}(\Delta)$ is the character of the symmetric group $S_{d}$ evaluated at a cycle type $\Delta$, and $\chi_{\lambda}$ ranges over the irreducible complex characters of $S_{d}$, labeled by partitions $\lambda=\left(\lambda_{1}, \ldots, \lambda_{\ell}\right)$. The convenient notion of normalized character, $\varphi_{\lambda}$, comes from [51, 1]. Each profile $\Delta^{(i)}$ is a partition of $d$ - the set of nonnegative non-increasing numbers $\left(d_{1}^{(i)}, d_{2}^{(i)}, \ldots\right)$, which describes the ramification over the point number $i$ on the base. The weights of all partitions involved in (1) are equal: $|\lambda|:=\sum_{j} \lambda_{j}=\left|\Delta^{(i)}\right|:=\sum_{j} d_{j}^{(i)}=d$. The number $\left|C_{\Delta}\right|$ is the number of elements in the cycle class $\Delta$ in $S_{d}$. The sum (1) runs over partitions of the weight $d$. We assume that $\varphi_{\lambda}(\Delta)$ vanishes in case $|\lambda| \neq|\Delta|$.

The Hurwitz numbers form a topological field theory [13. In the string theory applications the covering surface is the worldsheet of the string while the base surface is the target space. Hurwitz numbers are used in mathematical physics (for instance in [13]) and in algebraic geometry [37. A lot of interest and the developments in these studies appeared due to the work 15 which relates Hurwitz numbers to Gromov-Witten theory.

Our paper deals with enumeration of the covers of the projective plane $\mathbb{R P}^{2}$ (the case $\mathrm{E}=1$ in (1D)); the related Hurwitz numbers will be called projective. The projective Hurwitz numbers were introduced in Mednykh-Pozdnyakova work 43 and independently in the context of topological field theory in [5].

In this case we found that it is a different hierarchy of integrable equations which is related to the problem: this is the BKP hierarchy introduced by V.Kac and J. van de Leur in [32]1. In certain sense this hierarchy is very similar to the DKP one introduced in 31, however the difference between D and B types is crucial for the counting problem we need (see Remark 23 in the Appendix). Somehow the BKP hierarchy of Kac-van de Leur is not well-known, though it has applications to the famous orthogonal and symplectic ensembles 38 and some other models of random matrices and random partitions 39 , 54, 58. We are going to show that the BKP tau function of the hypergeometric type introduced in [58] generates Hurwitz numbers for covers of $\mathbb{R P}^{2}$. Up to an unimportant factor the BKP tau function of the hypergeometric type may be written as follows

$$
\tau^{\mathrm{B}}(N, n, \mathbf{p})=\sum_{\substack{\lambda \in \mathrm{P} \\ \ell(\lambda) \leq N}} s_{\lambda}(\mathbf{p}) c^{|\lambda|} \prod_{(i, j) \in \lambda} r(n+j-i)
$$

where $s_{\lambda}$ is the Schur function [40, related to a partition $\lambda=\left(\lambda_{1}, \ldots, \lambda_{\ell}\right), \ell(\lambda)$ denotes the number of nonvanishing parts of $\lambda, c$ is a parameter and $\mathrm{P}$ denotes the set of all partitions (in what follows we will omit to point out the summation range $\mathrm{P}$, having it in mind). The product in the right hand side ranges over all nodes of the Young diagram $\lambda, j$ is the column and $i$ is the row coordinate of the node of $\lambda$ depicted in English way where the diagonal spreads down and right from the origin. Two discrete parameters $N$ and $n$ and the set $\mathbf{p}=\left(p_{1}, p_{2}, \ldots\right)$ are called the BKP higher times 32$]^{2}$. We suppose that the tau function (3) is equal to 1 if $N=0$ and vanishes if $N<0 . r$ is an arbitrary chosen function of one variable, it will be specified later according to the needs of our work. The number $j-i$ is called the content of the node located at $i$-th row and $j$-th column of the Young diagram $\lambda$; the product over all nodes of the Young diagram in the right hand side of (3) is called content product (the generalized Pochhammer symbol). Content products play an essential role in the study of applications

\footnotetext{
${ }^{1}$ This BKP hierarchy was called "charged" and "fermionic" BKP hierarchy in 32. We call it "large" BKP hierarchy because it includes KP one and may be related 39] to the two-component KP. The "small" KP hierarchy, introduced in 31] is a subhierarchy in the KP one.

2 In the present paper we use the so-called power sums $p_{m}$ [40] as higher time variables rather than $\frac{1}{m} p_{m}$ as it is common in the soliton theory 31 .
} 
of the symmetric groups (for instance, see [19], [24], [23] and references therein). The special role of the content product in the study of Hurwitz numbers generated by KP hierarchy was observed and worked out in 20 .

In the present paper we chose two different types of parameterizations of the function $r$ which defines the content product in (3). The first is

$$
r(x)=\exp \sum_{m>0} \frac{1}{m} \zeta_{m} h^{m} x^{m}
$$

The second is

$$
r(x)=\mathrm{t}^{x \xi_{0}} \exp \sum_{m \neq 1} \frac{1}{m} \xi_{m} \mathrm{t}^{m x}
$$

The complex number $\mathrm{t}$ and sets $\left\{\zeta_{m}, m>0\right\}$ and $\left\{\xi_{m}, m \in \mathbb{Z}\right\}$ are free parameters. Similar to [51, 23] we introduce auxiliary parameters $c$ and $h$, the powers of $c$ count the degree of covering maps while the powers of the parameter $\frac{1}{h}$ which enters (4) count the Euler characteristic of the covers. In what follows we may put $c=1$ and $h=1$ in cases we are not interested in the degree and the Euler characteristic, and hope this does not produce a confusion. Everywhere if it is possible without a confusion we also avoid writing down the dependence of $r(x)$ and of $\tau(N, n, \mathbf{p})$ on $c, h, \zeta, \xi$ and other parameters to make formulae more readable.

Let us note that the usage of the parametrization I in the applications the content product was also considered in 29] in the study the combinatorial Hurwitz numbers by using of Cayley graphs and Jusys-Murphy elements as it was suggested by Canadien combinatorial school 21 and developed in 23.

One of the results of our paper is the explicit expressions of the content products parameterized by (41) and by (5) in terms of characters of symmetric group, see Propositions 2 and 3

Let us write down the answer for the case (II):

$$
\prod_{(i, j) \in \lambda} r(x+j-i)=\left.\mathrm{t}^{\xi_{0} x|\lambda|+\xi_{0} \varphi_{\lambda}(\Gamma)} \exp \sum_{m \neq 0} \frac{1}{m} \xi_{m} \mathrm{t}^{m x} D_{p_{1}} \log s_{\lambda}(\mathbf{p})\right|_{\mathbf{p}\left(0, \mathrm{t}^{m}\right)}, \quad|\Gamma|=|\lambda|=d
$$

Here first we apply the Euler operator $D_{p_{1}}=p_{1} \frac{\partial}{\partial p_{1}}$ to the Schur function $s_{\lambda}(\mathbf{p})$ where $\mathbf{p}=\left(p_{1}, p_{2}, \ldots\right)$, then evaluate the result at the point $\mathbf{p}=\mathbf{p}\left(0, \mathrm{t}^{m}\right)=\left(p_{1}\left(0, \mathrm{t}^{m}\right), p_{2}\left(0, \mathrm{t}^{m}\right), \ldots\right)$ where $p_{k}\left(0, \mathrm{t}^{m}\right)=$ $\left(1-\mathrm{t}^{m k}\right)^{-1}$. The partition $\Gamma$ is defined as follows. For $d \geq 2$ it is the partition $\left(1^{d-2} 2\right)$, it's length is $\ell(\Gamma)=d-1$. We choose the notation $\Gamma$ because the Young diagram of the partition $\left(1^{d-2} 2\right)$ resembles the capital Greek letter gamma. The cycle class labeled by $\Gamma$ in $S_{d}$ consists of all transpositions. We keep the notation $\Gamma$ also for the case $d \leq 1$, then $\Gamma=(d)$.

One can see that the content product for a partition $\lambda$ is expressed in terms of the Schur functions labeled by the same partition. Thanks to the characteristic map relation [40]

$$
s_{\lambda}(\mathbf{p})=\frac{\operatorname{dim} \lambda}{d !}\left(p_{1}^{d}+\sum_{\substack{\Delta \\ \Delta \neq 1}} \varphi_{\lambda}(\Delta) \mathbf{p}_{\Delta}\right)
$$

formula (6) produces series in $\varphi_{\lambda}$ which, in turn, due to the summation over partitions $\lambda$ in (3), allows us to consider (3) as the generating function for Hurwitz numbers (1). The content product (6) is expressed in terms of the Schur function and formula (7) exhibits the explicit dependence of the Schur functions on $\operatorname{dim} \lambda$. However in the expression for the content product (6) the dependence on $\operatorname{dim} \lambda$ disappears thanks to the logarithmic derivative of the Schur function. Then it follows that in the generating series (11) the exponent E (the Euler characteristic) is equal to 1. Therefore we obtain generating series for the projective Hurwitz numbers (to be precise - generating series for weighted sums of the projective Hurwitz numbers, where as we shall see later, the weights are defined by specifications of the parameters $\xi$, t).

The case (I) may be considered as the degeneration of the case (II) when $t \rightarrow 1$, therefore in this case series (3) also generate projective Hurwitz numbers.

Here and below, the notation $\mathbf{p}_{\Delta}$ serves for the product $p_{d_{1}} p_{d_{2}} \cdots$ where $d_{i}$ are the parts of the partition $\Delta: \Delta=\left(d_{1}, d_{2}, \ldots\right)$. 
Then the tau function (3) may be presented as

$$
\tau^{\mathrm{B}}(N, n, \mathbf{p})=\sum_{d \geq 0} c^{d} \sum_{\substack{\Delta \\|\Delta|=d}} \mathrm{H}_{r}(d ; \Delta) \mathbf{p}_{\Delta}, \quad \mathrm{H}_{r}(d ; \Delta)=\sum_{\substack{\lambda \\|\lambda|=d, \ell(\lambda) \leq N}} \frac{\operatorname{dim} \lambda}{d !} \varphi_{\lambda}(\Delta) \prod_{(i, j) \in \lambda} r(n+j-i)
$$

where (for $d \leq N) H_{r}(d ; \Delta)$ is a certain series in Hurwitz numbers which describe $d$-fold covers with the ramification $\Delta$ over a point, say, 0 of $\mathbb{R P}^{2}$ and ramifications over additional points which are determined by the choice of $r$, namely, of the parameters in (44) or in (5). We should keep in mind that in (3) it is only the part of sum over $\lambda$ conditioned by $|\lambda| \leq N$ generates Hurwitz numbers $H^{\mathrm{E}, \mathrm{F}}(d=|\lambda| ; \ldots)$. Thus to get Hurwitz number for the study of $d$-fold coverings one should work with series (3) conditioned by $N \geq d$. The same restriction we meet in Section 8 when consider integrals over $N \times N$ matrices which generate Hurwitz numbers.

Remark 1. As we see, the sum

$$
\sum_{\lambda} c^{|\lambda|} \prod_{(i, j) \in \lambda} r(n+j-i)
$$

also may be viewed as the generating function of the Hurwitz numbers when the base surface has Euler characteristic equal to zero (which either the torus, or the Klein bottle). In case of the specification (44) such sums may be related to the characters of the Lie algebra of differential operators on the circle as it was studied in [11.

Both choices of the content products, (41) and (5), contain the direct analogue of the Okounkov generating series [51, now, however for covers of $\mathbb{R P}^{2}$. It is enough to put $\zeta_{m}=0$ except $\zeta_{1}$ in (4), or, to put all $\xi_{m}=0$ except $\xi_{0}$ in (5).

Using respectively (4) and (5) we obtain two different types of generating functions for the projective Hurwitz numbers. The first one arising from (4) may be compared to the approach based on completed cycles developed in [52, [1] (where the $\mathbb{C P}^{1}$ case was studied). The second one, obtained from (5), is related to a ' $q$-deformation' of the previous case (where instead of $q$ we use the letter $\mathrm{t}$ ) which in turn may be compared to the approaches developed independently in [50] and in [29]. We will show that in the "t-deformed" (or, "trigonometric") case the Hall-Littlewood and the Macdonald polynomials naturally appear as weight functions in weighted sums of the projective Hurwitz numbers.

The structure of the paper is as follows. In Section 2 we explain the notion of Hurwitz numbers for Klein surfaces. In the Subsection 2.2 we present links between Hurwitz numbers with different Euler characteristics E of base surfaces. There we also explain the special role of the ramification described by the one row Young diagram $(d)$ (maximally ramified profile) in the enumeration of the $d$-fold covers presented in Proposition 1. It means that BKP hypergeometric function also generate Hurwitz numbers for the $d$-fold covers of any Klein surfaces in case at least one of the profiles is maximally ramified. In Section 3 we find the content products for cases (I) and (II) in terms of characters of the symmetric groups. The answers are respectively given by Propositions 2 and $3^{3}$ In Section 4 we introduce weighted sums of the projective Hurwitz numbers (which as we will show in further sections are generated by the BKP tau functions). For the weighting in particular we use the Macdonald, Jack and Hall-Littlewood polynomials which naturally appear via specifications of the parametrization of $r$. Now we are ready to use tau functions. In Section 5 we recall the notion of the BKP hierarchy and of the special family of the BKP tau functions called hypergeometric ones. We show that the BKP hypergeometric tau function may be obtained from the two-component KP hypergeometric tau function (which may be related to the semiinfinite TL equations) by an action of a special heat operator which was introduced in the Subsection 2.2. This action relates hierarchy serving respectively $\mathbb{C P}^{1}$ and $\mathbb{R P}^{2}$ Hurwitz counting problems. At the end of this section we how we get hypergeometric BKP tau functions with content products (4) and (5) in terms of an action of vertex operators on a special tau function $\tau_{1}^{\mathrm{B}}$. Section 6] is devoted to the set of examples of hypergeometric tau functions which are related to different choices of the parameters in (4) and (5). In Section 7.2 our main results are written down. We show that the tau function (3) together with either (4) or (5) generates projective Hurwitz numbers and weighted sums of the projective Hurwitz numbers. We show that, choosing the content product as in (5), sums weighted by Hall-Littlewood polynomials with the parameter $t$ appear naturally. We present the BKP tau functions which generate Hurwitz numbers with an arbitrary profile over 0 and additional branch points with two types of profiles: maximally ramified profiles and minimally ramified profiles (the simple branch points).

\footnotetext{
${ }^{3}$ The Propositions 2 is actually a new version of the known results presented in [1] about completed cycles, however we did not yet write down the correspondence in an explicit way.
} 
In the last Section 8 we present certain integrals over matrices, which generate projective Hurwitz numbers. Let us note that the well-known $\beta=2$ ensemble (or, the same, the unitary ensemble, or, the same, one-matrix model) counts both $\mathbb{C P}^{1}$ Hurwitz numbers and ribbon graphs with a given number of faces, vertices and edges, see [41, and as it was shown in [50] (see Section 6 there) the simplest way to get it is to present the one-matrix model as a hypergeometric tau function [25]. We do not manage to do the same in $\mathbb{R P}^{2}$ case. The analogues of the unitary ensemble are $\beta=1,4$ ensembles (the orthogonal and symplectic ones) which produce Feynman graphs, each one may be embedded either in orientable surface (in case it is a ribbon graph) or to non-orientable one (in case it is a ribbon graph with crosscaps). It was shown in 38 that partition functions of these ensembles are BKP tau functions. However the perturbation series written as series of the Schur functions (see [58) are not series we need 4 . To get the projective Hurwitz numbers we suggest another matrix integrals. These integrals in the integration measure contain the simplest BKP tau function $\tau_{1}^{B}$ which is widely used in our paper, see (18), (71), (75), (78) and (107) below. Such integral of a BKP tau function may be BKP tau function again but may be not a tau function, the last case occurs when the integral generate Hurwitz numbers with arbitrary profiles in two and more branch points. We also point out that the multiple usage of the BKP tau function $\tau_{1}^{\mathrm{B}}$ to deform integration measures of matrix integrals allows to get Hurwitz numbers related to arbitrary Euler characteristic of the base surface.

To end the introduction let us note that if in (3) we take $r$ as in (4) and choose $\mathbf{p}=(1,0,0, \ldots)$, then (3) is a discrete version of the partition function of the orthogonal ensemble of random matrices:

$$
\begin{gathered}
\tau^{\mathrm{B}}=\frac{1}{g(n) N !} \sum_{h_{1}, \ldots, h_{N} \geq 0} \prod_{i<j}\left|h_{i}-h_{j}\right| \prod_{i=1}^{N} \frac{e^{V\left(p^{*}, h_{i}\right)}}{h_{i} !} \\
V\left(p^{*}, x\right):=\sum_{m>0} \frac{1}{m} x^{m} p_{m}^{*}
\end{gathered}
$$

where as we shall see, the variables $\zeta$ and $\mathbf{p}^{*}$ are related via $V\left(\mathbf{p}^{*}, x-1\right)-V\left(\mathbf{p}^{*}, x\right)=V(\zeta, x)$. From 38 we know that (10) is the BKP tau function with the variables $\mathbf{p}^{*}$ playing the role of BKP higher times. The factor $g(n)$ is written down in the Appendix B

In a similar way we may obtain a discrete analogue of the circular $\beta=1$ ensemble choosing (5), see Remark 20 in Section 5 which proves that for a certain specification of $\mathbf{p}$ the series (3) is a BKP tau function with respect to the variables $\xi$.

The relation (10) may be interesting because $\beta=1$ ensembles generates Mobius graphs related to $n$-gulations of non-orientable surfaces, see [46] and references therein.

Now we shall study the above in detail. This paper is a development of our preprint [50].

\section{Hurwitz numbers}

\subsection{Definitions and examples}

For a partition $\Delta$ of a number $d=|\Delta|$ denote by $\ell(\Delta)$ the number of the non-vanishing parts. For the Young diagram corresponding to $\Delta$, the number $|\Delta|$ is the weight of the diagram and $\ell(\Delta)$ is the number of rows. Denote by $\left(d_{1}, \ldots, d_{\ell}\right)$ the Young diagram with rows of length $d_{1}, \ldots, d_{\ell}$ and corresponding partition of $d=\sum d_{i}$. We need the notion of the colength of a partition $\Delta$ which is $\ell^{*}(\Delta):=|\Delta|-\ell(\Delta)$.

Let us consider a connected compact surface without boundary $\Omega$ and a branched covering $f: \Sigma \rightarrow \Omega$ by a connected or non-connected surface $\Sigma$. We will consider a covering $f$ of the degree $d$. It means that the preimage $f^{-1}(z)$ consists of $d$ points $z \in \Omega$ except some finite number of points. This points are called critical values of $f$.

Consider the preimage $f^{-1}(z)=\left\{p_{1}, \ldots, p_{\ell}\right\}$ of $z \in \Omega$. Denote by $d_{i}$ the degree of $f$ at $p_{i}$. It means that in the neighborhood of $p_{i}$ the function $f$ is homeomorphic to $x \mapsto x^{d_{i}}$. The set $\left(d_{1} \ldots, d_{\ell}\right)$ is the partition of $d$, that is called topological type of $z$.

Fix now points $z_{1}, \ldots, z_{\mathrm{F}}$ and partitions $\Delta^{(1)}, \ldots, \Delta^{(\mathrm{F})}$ of $d$. Denote by

$$
\widetilde{C}_{\Omega\left(z_{1} \ldots, z_{\mathrm{F}}\right)}\left(d ; \Delta^{(1)}, \ldots, \Delta^{(\mathrm{F})}\right)
$$

\footnotetext{
${ }^{4}$ Recently appeared the paper [12] where the graph counting of $\beta=1,2$ ensembles was studied in details.
} 
the set of all branched covering $f: \Sigma \rightarrow \Omega$ with critical points $z_{1}, \ldots, z_{\mathrm{F}}$ of topological types $\Delta^{(1)}, \ldots, \Delta^{(\mathrm{F})}$.

Coverings $f_{1}: \Sigma_{1} \rightarrow \Omega$ and $f_{2}: \Sigma_{2} \rightarrow \Omega$ are called isomorphic if there exists an homeomorphism $\varphi: \Sigma_{1} \rightarrow \Sigma_{2}$ such that $f_{1}=f_{2} \varphi$. Denote by $\operatorname{Aut}(f)$ the group of automorphisms of the covering $f$. Isomorphic coverings have isomorphic groups of automorphisms of degree $|\operatorname{Aut}(f)|$.

Consider now the set $C_{\Omega\left(z_{1} \ldots, z_{\mathrm{F}}\right)}\left(d ; \Delta^{(1)}, \ldots, \Delta^{(\mathrm{F})}\right)$ of isomorphic classes in $\widetilde{C}_{\Omega\left(z_{1} \ldots, z_{\mathrm{F}}\right)}\left(d ; \Delta^{(1)}, \ldots, \Delta^{(\mathrm{F})}\right)$. This is a finite set. The sum

$$
H^{\mathrm{E}, \mathrm{F}}\left(d ; \Delta^{(1)}, \ldots, \Delta^{(\mathrm{F})}\right)=\sum_{f \in C_{\Omega\left(z_{1} \ldots, z_{\mathrm{F}}\right)}\left(d ; \Delta^{(1)}, \ldots, \Delta^{(\mathrm{F})}\right)} \frac{1}{|\operatorname{Aut}(f)|},
$$

don't depend on the location of the points $z_{1} \ldots, z_{\mathrm{F}}$ and is called Hurwitz number. Here $\mathrm{F}$ denotes the number of the branch points, and $\mathrm{E}$ is the Euler characteristic of the base surface.

Example. Let $f: \Sigma \rightarrow \mathbb{R P}^{2}$ be a covering without critical points. Then, if $\Sigma$ is connected, then $\Sigma=\mathbb{R P}^{2}, \operatorname{deg} f=1$ or $\Sigma=S^{2}, \operatorname{deg} f=2$. Therefore if $d=3$, then $\Sigma=\mathbb{R} \mathbb{P}^{2} \amalg \mathbb{R} \mathbb{P}^{2} \amalg \mathbb{R} \mathbb{P}^{2}$ or $\Sigma=\mathbb{R P}^{2} \amalg S^{2}$. Thus $H^{1,0}(3)=\frac{1}{3 !}+\frac{1}{2 !}=\frac{2}{3}$.

The Hurwitz numbers arise in different fields of mathematics: from algebraic geometry to integrable systems. They are well studied for orientable $\Omega$. In this case the Hurwitz number coincides with the weighted number of holomorphic branched coverings of a Riemann surface $\Omega$ by other Riemann surfaces, having critical points $z_{1}, \ldots, z_{\mathrm{F}} \in \Omega$ of the topological types $\Delta^{(1)}, \ldots, \Delta^{(\mathrm{F})}$ respectively. The well known isomorphism between Riemann surfaces and complex algebraic curves gives the interpretation of the Hurwitz numbers as the numbers of morphisms of complex algebraic curves.

Similarly, the Hurwitz number for a non-orientable surface $\Omega$ coincides with the weighted number of the dianalytic branched coverings of the Klein surface without boundary by another Klein surface and coincides with the weighted number of morphisms of real algebraic curves without real points [7, 47, 48, An extension of the theory to all Klein surfaces and all real algebraic curves leads to Hurwitz numbers for surfaces with boundaries may be found in [5, 49.

The Hurwitz numbers have a purely algebraic description. Any branched covering $f: \Sigma \rightarrow \Omega$ with critical points $z_{1}, \ldots, z_{\mathrm{F}} \in \Omega$ generates a homomorphism $\phi: \pi_{1}\left(u, \Omega \backslash\left\{z_{1}, \ldots z_{F}\right\}\right) \rightarrow S_{\Gamma}$, where $u$ is a point in $\Omega$, to the group of permutations of the set $\Gamma=f^{-1}(u)$ by the monodromy along contours of $\pi_{1}\left(u, \Omega \backslash\left\{z_{1}, \ldots z_{F}\right\}\right)$. Moreover, if $l_{i} \in \pi_{1}\left(u, \Omega \backslash\left\{z_{1}, \ldots z_{F}\right\}\right)$ is a contour around $z_{i}$, then the cyclic type of the permutation $\phi\left(l_{i}\right)$ is $\Delta^{(i)}$. Denote by

$$
\operatorname{Hom}_{\Omega}\left(d ; \Delta^{(1)}, \ldots, \Delta^{(\mathrm{F})}\right),
$$

the group of all homomorphisms $\phi: \pi_{1}\left(u, \Omega \backslash\left\{z_{1}, \ldots z_{F}\right\}\right) \rightarrow S_{\Gamma} \cong S_{d}$ with this property. Isomorphic coverings generate elements of $\operatorname{Hom}_{\Omega}\left(d ; \Delta^{(1)}, \ldots, \Delta^{(\mathrm{F})}\right)$ conjugated by $S_{d}$. Thus we construct the one-to-one correspondence between $C_{\Omega\left(z_{1} \ldots, z_{\mathrm{F}}\right)}\left(d ; \Delta^{(1)}, \ldots, \Delta^{(\mathrm{F})}\right)$ and the conjugated classes of $\operatorname{Hom}_{\Omega}\left(d ; \Delta^{(1)}, \ldots, \Delta^{(\mathrm{F})}\right)$.

Consider the last set in more details. Any $s \in S_{d}$ generates the interior automorphism $I_{s}(g)=s g s^{-1}$ of $S_{d}$. Therefore $S_{d}$ acts on $\operatorname{Hom}_{\Omega}\left(d ; \Delta^{(1)}, \ldots, \Delta^{(\mathrm{F})}\right)$ by $s(h)=I_{s} h$. The orbit of this action of $I=\left\{I_{s}\right\}$ corresponds to an equivalent class of coverings. Moreover, the group A $=\left\{s \in S_{d} \mid s(h)=h\right\}$ is isomorphic to the group Aut $(f)$, there the covering $f$ corresponds to the homomorphism $h$.

Consider the splitting $\operatorname{Hom}_{\Omega}\left(d ; \Delta^{(1)}, \ldots, \Delta^{(\mathrm{F})}\right)=\bigcup_{i=1}^{r} H_{i}$ on obits by $I$. Then the cardinality $\left|H_{i}\right|$ is $\frac{d !}{\left|\mathrm{A}\left(h_{i}\right)\right|}=\frac{d !}{\left|\operatorname{Aut}\left(f_{i}\right)\right|}$, where $h_{i} \in H_{i}$. On the other hand, the orbits $H_{i}$ are in the one-to-one correspondence with the classes of the coverings. Therefore $\frac{1}{d !}\left|\operatorname{Hom}_{\Omega}\left(d ; \Delta^{(1)}, \ldots, \Delta^{(\mathrm{F})}\right)\right|=\frac{1}{d !} \sum_{i=1}^{r}\left|H_{i}\right|=\sum_{i=1}^{r} \frac{1}{\left|\operatorname{Aut}\left(f_{i}\right)\right|}$ is the Hurwitz number $H_{\Omega}\left(d ; \Delta^{(1)} \ldots, \Delta^{(\mathrm{F})}\right)$.

Find now $\left|\operatorname{Hom}_{\Omega}\left(d ; \Delta^{(1)}, \ldots, \Delta^{(\mathrm{F})}\right)\right|$ in terms of the characters of $S_{d}$. Recall, that cyclic type of $s \in S_{d}$ is cardinalities $\Delta=\left(d_{1}, \ldots, d_{\ell}\right)$ of subsets, on which the permutation $s$ split the set $\{1, \ldots, d\}$. Any partition $\Delta$ of $d$ generates the set $C_{\Delta} \subset S_{d}$, consisting of permutations of cyclic type $\Delta$. The cardinality of $C_{\Delta}$ is equal to

$$
\left|C_{\Delta}\right|=\frac{|\Delta| !}{z_{\Delta}}, \quad z_{\Delta}=\prod_{i=1}^{\infty} i^{m_{i}} m_{i} !
$$


where $m_{i}$ denotes the number of parts equal to $i$ of the partition $\Delta$ (then the partition $\Delta$ is often denoted by $\left.\left(1^{m_{1}} 2^{m_{2}} \cdots\right)\right)$.

Moreover, if $s_{1}, s_{2} \in C_{\Delta}$, then $\chi\left(s_{1}\right)=\chi\left(s_{2}\right)$ for any complex character $\chi$ of $S_{d}$. Thus we can define $\chi(\Delta)$ for a partition $\Delta$, as $\chi(\Delta)=\chi(s)$ for $s \in C_{\Delta}$.

The Mednykh-Pozdnyakova-Gareth A. Jones formula [18, 37, 42, 43, says that

$$
\left|\operatorname{Hom}_{\Omega}\left(d ; \Delta^{(1)}, \ldots, \Delta^{(\mathrm{F})}\right)\right|=d ! \sum_{\lambda}\left(\frac{\operatorname{dim} \lambda}{d !}\right)^{\mathrm{E}} \prod_{i=1}^{\mathrm{F}}\left|C_{\Delta^{(i)}}\right| \frac{\chi\left(\Delta^{(i)}\right)}{\operatorname{dim} \lambda}
$$

where $\mathrm{E}=\mathrm{E}(\Omega)$ is the Euler characteristic of $\Omega$ and $\chi$ ranges over the irreducible complex characters of $S_{d}$, associated with Young diagrams of weight $d$. Thus we obtain (1).

In particulary for the projective plane $\mathbb{R P}^{2}$ we get the relation (10) where $\mathrm{E}=1$.

Example. Let $\mathrm{E}=1, \mathrm{~F}=0, d=3$. Then, $H^{1,0}(3)=\sum_{|\lambda|=3} \frac{\operatorname{dim} \lambda}{d !}=\frac{4}{6}=\frac{2}{3}$. In general for the unbranched covering of $\mathbb{R P}^{2}$ we get the following generating function (compare to (71) below)

$$
e^{\frac{c^{2}}{2}+c}=\sum_{d \geq 0} c^{d} H^{1,0}(d)
$$

The exponent reflects the fact that the connected unbranched covers of the projective plane may consist of either the projective plane (the term $c$ : 1-fold cover) or the Riemann sphere (2-fold cover, the term $\frac{c^{2}}{2}$ where 2 in the denominator is the order of the automorphisms of the covering by the sphere).

At the end we write down purely combinatorial definition of the projective Hurwitz numbers [43], [18. Let us consider the symmetric group $S_{d}$ and the equation

$$
R^{2} X_{1} \cdots X_{\mathrm{F}}=1, \quad R, X_{i} \in S_{d}, \quad X_{i} \in C_{\Delta^{(i)}}, i=1, \ldots, \mathrm{F}
$$

where $C_{\Delta^{(i)}}, i=1, \ldots, \mathrm{F}$ are the cycle classes of a given set of partitions $\Delta^{(i)}, i=1, \ldots, \mathrm{F}$ of a given weight $d$. Then $H^{1, \mathrm{~F}}\left(d ; \Delta^{(1)}, \ldots, \Delta^{(\mathrm{F})}\right)$ is the number of solutions to (14) divided over $d$ !. Say, for unbranched 3 -fold covering we get 4 solutions to $R^{2}=1$ in $S_{3}$ : the unity element and three transpositions. Thus $H^{1,0}=4: 3$ ! as we obtained in the Examples above. The number of solutions to $R^{2}=1$ in $S_{d}$ is given by (13).

\subsection{Remarks on Mednykh-Pozdnyakova-Gareth A. Jones character formula}

Let us start from the following preliminary

Remark 2. It follows from R.Dijkgraaf paper 13 that the Hurwitz numbers for closed orientable surfaces form $2 \mathrm{D}$ topological field theory. An extension of this result to the case of Klein surfaces (thus to orientable and non-orientable surfaces) was found in [5], theorem 5.2. (see also Corollary 3.2 in [6]) On the other hand MednykhPozdnyakova-Gareth A. Jones formula gives the description of the Hurwitz numbers in terms of characters of the symmetric groups. In this Subsection in fact we give the interpretation of axioms of the Klein topological field theory [5] for Hurwitz numbers in terms of characters of symmetric groups, this approach is different from [5].

(A) Let us present the following simple statement

\section{Lemma 1.}

$H^{\mathrm{E}+\mathrm{E}_{1}, \mathrm{~F}^{+} \mathrm{F}_{1}}\left(d ; \Delta^{(1)}, \ldots, \Delta^{\left(\mathrm{F}+\mathrm{F}_{1}\right)}\right)=\sum_{\Delta} \frac{d !}{\left|C_{\Delta}\right|} H^{\mathrm{E}+1, \mathrm{~F}+1}\left(d ; \Delta^{(1)}, \ldots, \Delta^{(\mathrm{F})}, \Delta\right) H^{\mathrm{E}_{1}+1, \mathrm{~F}_{1}+1}\left(d ; \Delta, \Delta^{(\mathrm{F}+1)}, \ldots, \Delta^{\left(\mathrm{F}_{1}\right)}\right)$

In particular

$$
H^{\mathrm{E}-1, \mathrm{~F}}\left(d ; \Delta^{(1)}, \ldots, \Delta^{(\mathrm{F})}\right)=\sum_{\Delta} H^{\mathrm{E}, \mathrm{F}+1}\left(d ; \Delta^{(1)}, \ldots, \Delta^{(\mathrm{F})}, \Delta\right) \chi(\Delta)
$$

where $\chi(\Delta)=\frac{d ! H^{1,1}(d ; \Delta)}{\left|C_{\Delta}\right|}$ are rational numbers explicitly defined by a partition $\Delta$ as follows

$$
\chi(\Delta)=\sum_{\substack{\lambda \\|\lambda|=|\Delta|}} \chi_{\lambda}(\Delta)=\left[\prod_{i>0, \text { even }} e^{\frac{i}{2} \frac{\partial^{2}}{\partial p_{i}^{2}}} \cdot p_{i}^{m_{i}} \prod_{i>0, \text { odd }} e^{\frac{i}{2} \frac{\partial^{2}}{\partial p_{i}^{2}}+\frac{\partial}{\partial p_{i}}} \cdot p_{i}^{m_{i}}\right]_{\mathbf{p}=0}
$$


where $\chi_{\lambda}(\Delta)$ is the character of the representation $\lambda$ of the symmetric group $S_{d}, d=|\lambda|$, evaluated on the cycle class $\Delta=\left(1^{m_{1}} 2^{m_{2}} \cdots\right)$.

As a Corollary we get that the Hurwitz numbers of the projective plane may be obtained from the Hurwitz numbers of the Riemann sphere while the Hurwitz numbers of the torus and of the Klein bottle (see (9) ) may be obtained from Hurwitz numbers of the projective plane.

First we prove the second equality in (17). It follows from the relations

$$
\begin{gathered}
e^{\sum_{i>0} \frac{i}{2} \frac{\partial^{2}}{\partial p_{i}^{2}}+\sum_{i>0, \text { odd }} \frac{\partial}{\partial p_{i}}}=\sum_{\lambda} s_{\lambda}(\tilde{\partial}) \\
{\left[s_{\lambda}(\tilde{\partial}) \cdot s_{\mu}(\mathbf{p})\right]_{\mathbf{p}=0}=\delta_{\lambda, \mu}, \quad p_{1}^{m_{1}} p_{2}^{m_{2}} \cdots=: p_{\Delta}=\sum_{\lambda} \chi_{\lambda}(\Delta) s_{\lambda}(\mathbf{p})}
\end{gathered}
$$

where $s_{\lambda}(\tilde{\partial})$ is $s_{\lambda}(\mathbf{p})$ where each $p_{i}$ is replaced by $i \frac{\partial}{\partial p_{i}}$. The heat operator in the left hand side of (18) plays an important role. The relations (19) may be found in 40]. The relation (18) is derived from the known relation

$$
\sum_{\lambda} s_{\lambda}(\mathbf{p}(\mathbf{x}))=\prod_{i<j} \frac{1}{1-x_{i} x_{j}} \prod_{i} \frac{1}{1-x_{i}}, \quad p_{m}(\mathbf{x}):=\sum_{i} x_{i}^{m}
$$

which also may be found in [40].

Equality (15) follows from the orthogonality relation for characters: $\sum_{\Delta}\left|C_{\Delta}\right| \chi_{\lambda}(\Delta) \chi_{\mu}(\Delta)=d ! \delta_{\mu, \lambda}$ where $|\mu|=|\lambda|=|\Delta|=d$ which yields $\sum_{\Delta} \varphi_{\lambda}(\Delta) \chi(\Delta)=d !(\operatorname{dim}(\lambda))^{-1}$. Then the formula (11) gives (15).

In (73) below we shall see that the heat operator which enters formula (17) also links solutions of 2KP (TL) and BKP hierarchies.

(B) Another remark is as follows.

Let us use the so-called Frobenius notation [40] for a partition $\lambda: \lambda=\left(\alpha_{1}, \ldots, \alpha_{\kappa} \mid \beta_{1}, \ldots, \beta_{\kappa}\right), \alpha_{1}>$ $\cdots>\alpha_{\kappa} \geq 0, \beta_{1}>\cdots>\beta_{\kappa} \geq 0$. The integer $\kappa=\kappa(\lambda)$ denotes the length of the main diagonal of the Young diagram $\lambda$, the length of $\lambda$ is denoted by $\ell(\lambda)$.

Lemma 2. The normalized character labeled by $\lambda$ evaluated at the cycle $(d)$ (as usual $d=|\lambda|$ ) vanishes if $\kappa(\lambda)>1$, moreover

$$
\varphi_{\lambda}((d))=(-1)^{\ell(\lambda)+1}\left(\frac{d !}{\operatorname{dim} \lambda}\right) \frac{1}{d} \delta_{1, \kappa(\lambda)}
$$

For the proof first we notice that the Schur function of a one-hook partition, say, $\left(\alpha_{i} \mid \beta_{j}\right)$ is of form

$$
s_{\left(\alpha_{i} \mid \beta_{j}\right)}(\mathbf{p})=\frac{1}{d}(-1)^{\beta_{j}} p_{\alpha_{i}+\beta_{j}+1}+\cdots
$$

where dots denote terms which do not depend on $p_{a}, a \geq \alpha_{i}+\beta_{j}+1$ (this fact may be derived, say, from the Jacobi-Trudi formula $\left.s_{\lambda}(\mathbf{p})=\operatorname{det} s_{\left(\lambda_{i}-i+j\right)}(\mathbf{p})\right)$. Then from the Giambelli identity $s_{\lambda}(\mathbf{p})=\operatorname{det} s_{\left(\alpha_{i} \mid \beta_{j}\right)}(\mathbf{p})$ it follows that $s_{\lambda}$ does not depend on $p_{a}, a>\alpha_{1}+\beta_{1}+1$. Thus it does not depend on $p_{a}, a \geq d>\alpha_{1}+\beta_{1}+1$ in case $\kappa(\lambda)>1$. Due to the character map relation it means that $\varphi_{\lambda}((d))=0$ if $\kappa(\lambda)>1$. In case of a one-hook partition $\lambda=\left(\alpha_{1} \mid \beta_{1}\right)$ we have $\alpha_{1}+\beta_{1}+1=d$ and the character map formula (7) yields

$$
s_{\left(\alpha_{1} \mid \beta_{1}\right)}(\mathbf{p})=\frac{\operatorname{dim} \lambda}{d !}\left(p_{d} \varphi_{\lambda}((d))+\cdots\right)
$$

where dots denote terms which does not depend on $p_{d}$. We compare last two formulae and get (21).

Relation (21) allows to equate Hurwitz numbers related to different Euler characteristics of base Klein surfaces if in both cases there are nonvanishing numbers of ramification profiles $(d)$. Namely, the Mednykh-Pozdnyakova-Gareth A. Jones character formula (1) yields

Proposition 1. For any natural number $g$

$$
H^{\mathrm{E}-2 g, \mathrm{~F}+1}\left(d ; \Delta^{(1)}, \ldots, \Delta^{(\mathrm{F})},(d)\right)=d^{2 g} H^{\mathrm{E}, \mathrm{F}+2 g+1}(d ; \Delta^{(1)}, \ldots, \Delta^{(\mathrm{F})},(d), \underbrace{(d), \ldots,(d)}_{2 g})
$$


First it was proven by Zagier (for the case of even E), see Appendix A in [37]. We get it in a different way.

For $d$-fold covers we shall call a branch point maximally ramified in case its ramification profile is $(d)$.

Remark 3. Notice that the presence of the profile $(d)$ means that the Hurwitz numbers of the connected and disconnected covering are equal: $H_{\text {connected }}^{\mathrm{E}, \mathrm{F}}(d ;(d), \ldots)=H^{\mathrm{E}, \mathrm{F}}(d ;(d), \ldots)$ where dots denote the same set of ramification profiles.

Remark 4. In the Appendix A by Zagier in 37] the polynomial $\mathrm{R}_{\Delta}(\mathrm{q}):=\frac{\prod_{i=1}^{\ell \Delta}\left(1-\mathrm{q}^{d_{i}}\right)}{1-\mathrm{q}}=: \sum_{r}(-1)^{r} \mathrm{q}^{r} \chi_{r}(\Delta)$ was studied. It was shown that $\chi_{r}(0 \leq r \leq d-1)$ is the character of the irreducible representation of $S_{d}$ given by $\chi_{r}(g)=\operatorname{tr}\left(g, \pi_{r}\right), g \in S_{d}, \pi_{r}=\wedge^{r}\left(\mathrm{St}_{d}\right)$. Here $\mathrm{St}_{d}$ is the vector space $\left\{\left(x_{1}, \ldots, x_{d}\right) \in \mathbb{C}^{d} \mid x_{1}+\cdots+x_{d}=0\right\}$ and $S_{d}$ acts by permutations of the coordinates. We can show that $\chi_{r}$ coincides with $\chi_{\lambda}$ where $\lambda=(d-r \mid r)$. To do it let us consider the Schur function $s_{\lambda}(\mathbf{p}(\mathbf{q}, 0))$ where $p_{m}(\mathbf{q}, 0):=1-\mathrm{q}^{m}$ and $\lambda$ is not yet fixed. We get

$$
s_{\lambda}(\mathbf{p})=(-1)^{\ell(\lambda)-1}(1-\mathbf{q}) \mathbf{q}^{\ell(\lambda)-1} \delta_{\kappa(\lambda), 1},
$$

see [40]. In the last relation denote $k=\ell(\lambda)-1$, then $\lambda=(d-k \mid k)$. On the other hand formula (7) says

$$
s_{(d-k \mid k)}(\mathbf{p}(\mathrm{q}, 0))=(1-\mathrm{q}) \sum_{\Delta} \frac{\left|C_{\Delta}\right|}{d !} \chi_{(d-k \mid k)}(\Delta) \mathrm{R}_{\Delta}(\mathrm{q})=(1-\mathrm{q}) \sum_{r=0}^{d-1}(-\mathrm{q})^{r} \sum_{\Delta} \frac{\left|C_{\Delta}\right|}{d !} \chi_{(d-k \mid k)}(\Delta) \chi_{r}(\Delta)
$$

Compare last relations. The orthogonality of characters results to $\chi_{k}=\chi_{(d-k \mid k)}$. It means that in the presence of a maximally ramified branch point the summation range in (1) is restricted to one-hook partitions $\lambda$.

Let us also note that the polynomials $\mathrm{R}_{\Delta}$ are related to ramification weights (47) below.

In what follows we shall see that tau functions generate Hurwitz numbers containing the maximally ramified branch points.

\section{Content products}

The content product which enters (3) may be written in form of generalized Pochhammer symbol

$$
\prod_{(i . j) \in \lambda} r(x+j-i)=\prod_{i=1}^{\ell(\lambda)} r_{\lambda_{i}}(x-i+1)
$$

where $r_{n}(x):=r(x) r(x+1) \cdots r(x+n-1)$, and also in form of a sort of Boltzmann weight

$$
\prod_{(i . j) \in \lambda} r(x+j-i)=e^{-U_{\lambda}(x)}:=\prod_{i=1}^{\ell(\lambda)} e^{U_{h_{i}(0)+x}-U_{h_{i}(\lambda)+x}}=\prod_{i=1}^{\kappa(\lambda)} e^{U_{\alpha_{i}+x}-U_{-\beta_{i}+x}}
$$

$\alpha_{i}, \beta_{i}, i=1, \ldots, \kappa$ are the Frobenius coordinates of the partition $\lambda, \lambda=\left(\alpha_{1}, \ldots \alpha_{\kappa} \mid \beta_{1}, \ldots, \beta_{\kappa}\right)(\kappa=\kappa(\lambda)$ in the length of the main diagonal of the Young diagram of the partition $\lambda$ ),

$$
h_{i}(\lambda):=\lambda_{i}-i, \quad r(x)=: \exp \left(U_{x-1}-U_{x}\right)
$$

The numbers $U_{x}$ may be fixed by $U_{x_{0}}=0$ with a chosen $x_{0}$. In the present paper $U_{x}$ is chosen either as $V(\zeta, x)$ (the parametrization I) or as $V\left(\xi, \mathrm{t}^{x}\right)+\xi_{0} \log \mathrm{t}$ (the parametrization II).

\subsection{Parametrization I}

Let us consider the sums of all normalized characters $\varphi_{\lambda}$ evaluated on partitions $\Delta$ with a given weight $d, d=|\lambda|=|\Delta|$ and a given length $\ell(\Delta)=d-k$ :

$$
\phi_{k}(\lambda):=\sum_{\substack{\Delta \\ \ell(\Delta)=d-k}} \varphi_{\lambda}(\Delta), \quad k=0, \ldots, d-1
$$


Remark 5. Let us note that $\phi_{0}(\lambda)=1$. There are two other special cases when the sum of normalized characters (26) contains a single term:

(a) $\phi_{1}(\lambda)=\varphi_{\lambda}(\Gamma), \Gamma=\left(1^{d-2} 2\right)$ (for $\left.d>1\right) . \phi_{1}(\lambda)=\varphi_{\lambda}(\Gamma)$ which is related to the minimally ramified profile: the profile with the colength equal to 1 . This is the profile of the simple branch point, simple branch points are of main interest in many applications 13 .

(b) $\phi_{d-1}(\lambda)=\varphi_{\lambda}((d))$ which is related to the cyclic profile which describes the maximally ramified profile (this profile plays a specific role, see Proposition 1).

In what follows we shall use sums $\phi_{k}$ as building blocks to construct weighted sums of the Hurwitz numbers (see for instance (60) below). Then the cases (a),(b) produce not the weighted sums but Hurwitz numbers themselves (see (98) below).

Remark 6. The quantity $d-\ell(\lambda)$ which is used in the definition (26) is called the colength of a partition $\lambda$ and will be denoted by $\ell^{*}(\lambda)$. The colength enters the so-called Riemann-Hurwitz formula which relates the Euler characteristic of a base surface, $\mathrm{E}$, to the Euler characteristic of it's $d$-branched cover, $\mathrm{E}^{\prime}$ as follows

$$
\mathrm{E}^{\prime}-d \mathrm{E}+\sum_{i} \ell^{*}\left(\Delta^{(i)}\right)=0
$$

where the sum ranges over all branch points.

Let us introduce

$$
\operatorname{deg} \phi_{k}(\lambda)=k
$$

This degree is equal to the colength of ramification profiles in formula (26), and due to Remark 6 it will be important later to define the Euler characteristic of the covering surfaces in the parametrization I cases.

Next we need

Lemma 3. The power sums of the contents of all nodes of a Young diagram $\lambda$ may be expressed in terms of the normalized characters and ratios of the Schur functions, and are polynomials in the variables $\phi_{k}, k=1,2, \ldots$ :

$$
\begin{aligned}
\Phi_{m}(\lambda) & :=\sum_{(i . j) \in \lambda}(j-i)^{m}= \\
& =\frac{m}{2 \pi i} \oint a^{m} \prod_{k=1}^{m}\left(1+\sum_{\Delta}\left(e^{2 \pi i \frac{k}{m}} a\right)^{-\ell^{*}(\Delta)} \varphi_{\lambda}(\Delta)\right) \frac{d a}{a} \\
& =(-1)^{m+1} m \frac{1}{2 \pi i} \oint a^{m} \log \frac{s_{\lambda}(\mathbf{p}(a))}{s_{\lambda}\left(\mathbf{p}_{\infty}\right)} \frac{d a}{a} \\
& =(-1)^{m+1} m \frac{1}{2 \pi i} \oint a^{m} \log \left(1+\sum_{k=1}^{d-1} a^{-k} \phi_{k}(\lambda)\right) \frac{d a}{a} \\
& =m \sum_{\substack{\mu \\
|\mu|=m, \mu_{1}<d}}(-1)^{\ell^{*}(\mu)}(\ell(\mu)-1) ! \frac{\phi_{\mu}(\lambda)}{\operatorname{Aut} \mu}
\end{aligned}
$$

where $m \geq 0,|\lambda|=|\Delta|$ and $\ell^{*}(\mu):=|\mu|-\ell(\mu)$ is the colength of the partition $\mu$.

$$
\phi_{\mu}(\lambda):=\prod_{i=k}^{d-1}\left(\phi_{k}(\lambda)\right)^{m_{k}}=\prod_{i=1}^{\ell(\mu)} \phi_{\mu_{i}}(\lambda), \quad \mu=\left(1^{m_{1}} \ldots(d-1)^{m_{d-1}}\right)=\left(\mu_{1}, \ldots, \mu_{\ell}\right)
$$

In (30) $\mathbf{p}(a)=(a, a, \ldots)$ and $\mathbf{p}_{\infty}=(1,0,0, \ldots)$, and in (32) Aut $\mu=\prod_{i=1}^{\ell(\mu)} m_{i} !$, $m_{i}$ denotes the number of times a part $i$ enters the partition $\mu=\left(1^{m_{1}} 2^{m_{2}} \cdots\right)$.

As we see from (32)-33) each integer $\Phi_{m}$ is a quasihomogeneous polynomial in the rational numbers $\phi_{k}$, and according to (27) we assign the degree as follows:

$$
\operatorname{deg} \Phi_{m}(\lambda)=m
$$


Let us write down the first three $\Phi_{m}(\lambda)$ for $|\lambda|=d \geq 4$ in terms of normalized characters $\varphi_{\lambda}$ using (32), (33), (26). We obtain

$$
\begin{gathered}
\Phi_{1}(\lambda)=\varphi_{\lambda}(\Gamma), \quad \Phi_{2}(\lambda)=\left(\varphi_{\lambda}(\Gamma)\right)^{2}-2 \varphi_{\lambda}\left(\left(1^{d-4} 2^{2}\right)\right)-2 \varphi_{\lambda}\left(\left(1^{d-3} 3^{1}\right)\right), \quad \Phi_{3}(\lambda)=\left(\varphi_{\lambda}(\Gamma)\right)^{3}- \\
3 \varphi_{\lambda}(\Gamma)\left(\varphi_{\lambda}\left(\left(1^{d-4} 2^{2}\right)\right)+\varphi_{\lambda}\left(\left(1^{d-3} 3^{1}\right)\right)\right)+3 \varphi_{\lambda}\left(\left(1^{d-4} 4^{1}\right)\right)+3 \varphi_{\lambda}\left(\left(1^{d-5} 2^{1} 3^{1}\right)\right)+3 \varphi_{\lambda}\left(\left(1^{d-6} 2^{3}\right)\right)
\end{gathered}
$$

Assume $d>2, m>2$. As one can see from (32), (33), (26) each $\Phi_{m}(\lambda)$ is of form $\left(\varphi_{\lambda}(\Gamma)\right)^{m}+\cdots$ where by dots we denote the contribution of cyclic classes marked by partitions, say $\Delta$, whose length $\ell(\Delta)$ belong either to the interval $[d-2, d-m]$ if $m<d$, or to the interval $[d-2,1]$ if $m \geq d$.

The proof that the right hand side of (28) is equal to (29) is based on two relations

$$
\lim _{n \rightarrow \infty} \prod_{k=1}^{m}\left(1-n^{-\frac{1}{m}} e^{2 \pi i \frac{k}{m}} x\right)^{n}=e^{-x^{m}}
$$

and

$$
\prod_{(i . j) \in \lambda}(a+j-i)=a^{|\lambda|}\left(1+\sum_{\Delta} \varphi_{\lambda}(\Delta) a^{\ell(\Delta)-|\lambda|}\right)=a^{|\lambda|}\left(1+\sum_{k=1}^{d-1} \phi_{k}(\lambda) a^{-k}\right)
$$

which may be obtained from relations in [40. This relation is important and will be further exploited to get Hurwitz numbers and weighted sums of Hurwitz numbers.

The proof that the right hand side of (28) is equal (31) follows from (36):

$$
\prod_{i, j \in \lambda} e^{-\sum_{m>0} \frac{1}{m}(-a)^{-m}(j-i)^{m}}=\prod_{i, j \in \lambda}\left(1+\frac{j-i}{a}\right)=1+\sum_{k=1}^{d-1} \phi_{k}(\lambda) a^{-k}
$$

Remark 7. By comparing the first and the last terms in (36) one can conclude that

$$
\phi_{k}(\lambda)=0, \quad \text { if } k>d-\kappa(\lambda)
$$

where $\kappa(\lambda)$ in the length of the main diagonal of the Young diagram of the partition $\lambda$. Now take $k=d-1$ as in (b) of the Remark 5 Then it follows that $\varphi_{\lambda}((d))$ is non-vanishing only for one-hook Young diagrams $\lambda=\left(d-a, 1^{a}\right), a=0,1, \ldots, d$.

Remark 8. It follows from the relation (36) that the following sum vanishes

$$
a^{d}\left(1+\sum_{k=1}^{d-1} \phi_{k}(\lambda) a^{-k}\right)=0
$$

if $a$ is integer and an addition if $-\lambda_{1}<a<\ell(\lambda)$.

Remark 9. From (36) we see that

where $\Phi(\lambda)=\left(\Phi_{1}(\lambda), \Phi_{2}(\lambda), \ldots\right)$

$$
s_{\left(1^{k}\right)}(\Phi(\lambda))=\phi_{k}(\lambda)
$$

Proposition 2. Let

$$
r(\zeta, h ; x)=\exp V(\zeta, h x)
$$

where $\zeta$ is the infinite set of parameters $\zeta=\left(\zeta_{1}, \zeta_{2}, \ldots\right)$ and $V$ is defined by (11). Then the related content product may be expressed in terms of characters in the following explicit way

$$
\prod_{(i . j) \in \lambda} r(\zeta, h ; j-i)=\exp \sum_{m>0} \frac{1}{m} h^{m} \zeta_{m} \Phi_{m}
$$

From (24) we get

$$
\prod_{(i . j) \in \lambda} r(\zeta, j-i)=\prod_{i=1}^{\kappa(\lambda)} e^{V\left(\mathbf{p}^{*}, \alpha_{i}\right)-V\left(\mathbf{p}^{*},-\beta_{i}-1\right)}=\prod_{i=1}^{L} e^{V\left(\mathbf{p}^{*}, h_{i}(\lambda)\right)-V\left(\mathbf{p}^{*}, 0\right)}
$$

where $h_{i}(\lambda)=\lambda_{i}-i$ and $\alpha_{i}, \beta_{i}$ are Frobenius coordinates of the partition $\lambda=(\alpha \mid \beta)$. Then the variables $\mathbf{p}^{*}=\left(p_{1}^{*}, p_{2}^{*}, \ldots\right)$ are related to the variables $\zeta$ by the triangle transformation given by

$$
V(\zeta, x)=V\left(\mathbf{p}^{*}, x-1\right)-V\left(\mathbf{p}^{*}, x\right)
$$

In particular we get the discrete version of the orthogonal ensemble given by (10). 
Remark 10. With the help of (40) the Proposition 2 may be related to the well-known results [52, [1] on Hurwitz numbers and the completed cycles as follows. In [1] the generation function for Hurwitz numbers of covers of $\mathbb{C P}^{1}$ in form

$$
\tau^{\mathrm{TL}}\left(\mathbf{p}^{(1)}, \mathbf{p}^{(2)} \mid \mathbf{p}^{*}\right)=\sum_{\lambda} e^{\sum_{m>0} \frac{1}{m} p_{m}^{*} \mathrm{C}_{\lambda}(m)} s_{\lambda}\left(\mathbf{p}^{(1)}\right) s_{\lambda}\left(\mathbf{p}^{(2)}\right)
$$

was studied and identified with a specification of the KP hypergeometric tau function [36, [57. The exponential prefactor in this KP hypergeometric tau function coincides with the right hand side of (40). Then it follows from (41) that

$$
\sum_{i=1}^{\ell(\lambda)}\left(\left(\lambda_{i}-i\right)^{m}-(-i)^{m}\right)=: \mathrm{C}_{\lambda}(m)=\sum_{k=1}^{m-1} \frac{(-1)^{m-k}}{(m-k) !} \frac{(m-1) !}{(k-1) !} \Phi_{k}(\lambda)
$$

Further remarks on (42):

Remark 11. (A) Let $\mathbf{p}^{(1)}=\mathbf{p}^{(2)}=(1,0,0, \ldots)$ in (42). Then the variables $\mathbf{p}^{*}$ may be identified with the $\mathrm{KP}$ higher times because the expression (42) yields a discrete version of the one-matrix model (the unitary ensemble), quite similarly to (10) which describes a discrete model the orthogonal ensemble. (B) If we choose $\mathbf{p}^{(1)}=\mathbf{p}^{(2)}=\mathbf{p}(0, \mathrm{t})$ (the notation see in the Introduction) and specify $p_{m}^{*}$ we obtain the partition functions of the $U(N)$ Chern-Simons model on $S^{3}$ with the coupling constant $g_{s}=-\log \mathrm{t}\left[8\right.$. (C) Let us take $\mathbf{p}^{(2)}=\mathbf{p}(0, \mathrm{t})$ and $p_{m}^{*}=0, m>2$, then the right hand side of (42) generates Marino-Vafa relations for Hodge integrals 62 (where $p, \lambda \tau$ and $\lambda$ are respectively $\mathbf{p}^{(1)}, p_{2}^{*}$ and $\sqrt{-1} \log \mathrm{t}$ in our notations). (D) It was first noticed in 36 ] (see also [59]) that for the choice $\mathbf{p}^{(1)}=(1,0,0, \ldots), p_{m}^{(2)}=\sum x_{i}^{m}$ the series [42) is a discrete version of the Kontsevich model:

$$
\tau^{\mathrm{TL}}\left(\mathbf{x}, \mathbf{p}^{*}\right)=\frac{1}{N !} \sum_{h_{1}, \ldots, h_{N}} \prod_{i<j}\left(h_{i}-h_{j}\right) \prod_{i=1}^{N} \frac{1}{h_{i} !} e^{V\left(\mathbf{p}^{*}, h_{i}\right)+\mathrm{L}_{i} h_{i}}, \quad x_{i}=e^{\mathrm{L}_{i}}
$$

\subsection{Parametrization II.}

If $j-i$ is the content of the node of $\lambda$ the number $\mathrm{t}^{j-i}$ is called the quantum content of the node.

Lemma 4. The power sums of the quantum contents $t^{j-i}$ of all nodes of the Young diagram $\lambda$ is expressed in terms of the parts of $\lambda$, Schur functions and the normalized characters $\varphi_{\lambda}$ as follows

$$
\begin{aligned}
T_{\lambda}(t) & :=\sum_{(i . j) \in \lambda} t^{j-i} \\
& =\sum_{i=1}^{\ell(\lambda)} t^{1-i} \frac{1-t^{\lambda_{i}}}{1-t}=\frac{t}{t-1} \sum_{i=1}^{\ell(\lambda)}\left(t^{h_{i}(\lambda)}-t^{h_{i}(0)}\right) \\
& =\left.p_{1} \frac{\partial}{\partial p_{1}} \log s_{\lambda}(\mathbf{p})\right|_{\mathbf{p}=\mathbf{p}(0, t)} \\
& =\frac{d+\sum_{\Delta}^{\prime} m_{1}(\Delta) A_{\Delta}(\lambda, t)}{1+\sum_{\Delta}^{\prime} A_{\Delta}(\lambda, t)}, \quad A_{\Delta}(\lambda, t)=\varphi_{\lambda}(\Delta) \frac{(1-t)^{d}}{\prod_{j=1}^{\ell(\Delta)}\left(1-t^{d_{j}}\right)}
\end{aligned}
$$

where $h_{i}(\lambda)=\lambda_{i}-i,|\lambda|=|\Delta|=d, \sum^{\prime}$ denotes the sum over all partitions except the partition $\left(1^{d}\right)$. The partition $\Delta$ is written either as $\left(d_{1}, \ldots, d_{\ell(\Delta)}\right)$ or as $\left(1^{m_{1}} 2^{m_{2}} \ldots\right), m_{i}=m_{i}(\Delta)$ denotes the number of parts of $\Delta$ equal to $i$. In formula (45) first we take the derivative with respect to $p_{1}$, then evaluate the power sum variables $\mathbf{p}$ as $\mathbf{p}=\mathbf{p}\left(0, t^{m}\right)=\left(p_{1}, p_{2}, \ldots\right)$ where $p_{k}=p_{k}\left(0, t^{m}\right)=\left(1-t^{k m}\right)^{-1}$.

The proof is similar to the previous case but instead of (36) we use another relation:

$$
\prod_{(i . j) \in \lambda} \frac{1-\mathrm{qt}^{j-i}}{1-\tilde{\mathrm{q}} \mathrm{t}^{j-i}}=\frac{s_{\lambda}(\mathbf{p}(\mathrm{q}, \mathrm{t}))}{s_{\lambda}(\mathbf{p}(\tilde{\mathrm{q}}, \mathrm{t}))}=\left(\frac{1-\mathrm{q}}{1-\tilde{\mathrm{q}}}\right)^{|\lambda|} \frac{1+\sum_{\Delta}^{\prime} \varphi_{\lambda}(\Delta) w(\Delta, \mathrm{q}, \mathrm{t})}{1+\sum_{\Delta}^{\prime} \varphi_{\lambda}(\Delta) w(\Delta, \tilde{\mathrm{q}}, \mathrm{t})}
$$

where $\mathbf{p}(\mathbf{q}, \mathbf{t})=\left(p_{1}(\mathbf{q}, \mathrm{t}), p_{2}(\mathbf{q}, \mathrm{t}), \ldots\right)$

$$
p_{m}(\mathrm{q}, \mathrm{t})=\frac{1-\mathrm{q}^{m}}{1-\mathrm{t}^{m}}
$$


and

$$
w(\Delta, \mathrm{q}, \mathrm{t})=\frac{(1-\mathrm{t})^{d}}{(1-\mathrm{q})^{d}} \prod_{i=1}^{\ell(\Delta)} \frac{1-\mathrm{q}^{d_{i}}}{1-\mathrm{t}^{d_{i}}}
$$

may be called q, t-ramification weight. We have $w\left(\Delta, e^{a h}, e^{h}\right) \rightarrow a^{\ell^{*}(\Delta)}$ as $h \rightarrow 0$. Equation (47) can be easily obtained from known relations presented in 40 .

For the proof we put $\tilde{\mathrm{q}}=0$ and replace $\mathrm{q} \rightarrow \frac{\mathrm{q}}{n}$ and consider the $n$-th power of (47) getting (46) from the right hand side of (47) where we insert (49). Then (45) follows from (46).

Remark 12. Apart from relations (43)-(46) one may also write

$$
T_{\lambda}\left(\mathrm{t}^{m}\right)=\frac{1}{2 \pi i} \oint \mathrm{q}^{-1-m} \log \frac{s_{\lambda}(\mathbf{p}(\mathbf{q}, \mathrm{t}))}{s_{\lambda}(\mathbf{p}(0, \mathrm{t}))} d \mathbf{q}, \quad m>0
$$

which is the analogue of (31).

Remark 13. We get $\Phi_{m}(\lambda)=\left.\left(\mathrm{t} \frac{\partial}{\partial t}\right)^{m} \cdot T_{\lambda}(\mathrm{t})\right|_{\mathrm{t}=1}$.

Proposition 3. Let

$$
r(\xi, x \mid t)=e^{V\left(\xi_{+}, t^{x}\right)+\xi_{0} x \log t+V\left(\xi_{-}, t^{-x}\right)}=e^{\sum_{m \neq 0} \frac{1-t^{m}}{m t^{m}} p_{m}^{*} t^{m x}+\xi_{0} x \log t}
$$

where $\xi$ is the collection of parameters $\xi_{0}$ and $\xi_{ \pm}=\left(\xi_{ \pm 1}, \xi_{ \pm 2}, \ldots\right)$, and where $V$ is defined by (11). Then

$$
\begin{gathered}
\prod_{(i . j) \in \lambda} r(\xi, x+j-i \mid t)=e^{\xi_{0}\left(\varphi_{\lambda}(\Gamma)+|\lambda| x\right) \log t+\sum_{m \neq 0} \frac{1}{m} \xi_{m} t^{m x} T_{\lambda}\left(t^{m}\right)}= \\
=\prod_{i=1}^{\ell(\lambda)} e^{\frac{\xi_{0} \log t}{2}\left(\left(x+h_{i}(\lambda)\right)^{2}+\left(x+h_{i}(\lambda)\right)-\left(x+h_{i}(0)\right)^{2}-\left(x+h_{i}(0)\right)\right)+\sum_{m \neq 0} \frac{1}{m} p_{m}^{*}\left(t^{\left(h_{i}(\lambda)+x\right) m}-t^{\left(h_{i}(0)+x\right) m}\right)}
\end{gathered}
$$

where $p_{m}^{*}=\xi_{m} \frac{t^{m}}{t^{m}-1}, h_{i}(\lambda)=\lambda_{i}-i, h_{i}(0)=-i$.

Remark 14. The right hand side of (47) may be obtained as the specification of parameters: $x=0, \xi_{m}=0$, $m \leq 0$, and $\xi_{m}=\tilde{\mathrm{q}}^{m}-\mathrm{q}^{m}, m>0$ in (51). Relation (47) is of use to get Hurwitz numbers in special cases. However one needs to explain how he would treat the denominator in the right hand side. Among others consider three different ways to do it:

(A) Let us fix $\tilde{\mathrm{q}}$. Then $w(\Delta, \tilde{\mathrm{q}}, \mathrm{t})$ tends to zero if $\mathrm{t} \rightarrow 1$ for $\Delta \neq\left(1^{d}\right)$. It allows to developed the denominator in the right hand side of (47) and also $T_{\lambda}(\mathrm{t})$ of (46) in Taylor series in the normalized characters of $S_{d}$ for $\mathrm{t}$ close to 1 . The limit $t \rightarrow 1$ returns us to the case studied in the Subsection 3.1

There are also two different limiting procedures which allows to get rid of the infinite sum arising from the character expansion of $s_{\lambda}(\mathbf{p}(\tilde{\mathrm{q}}, \mathrm{t}))$ in the denominator of the right hand side of 447) in case the leading term in the denominator is the term with $\Delta=(d)$ :

(B) The second one is based on taking $\tilde{q}$ to be close to 1 :

Lemma 5. Let $\epsilon$ be a small parameter. Then

$$
\varphi_{\lambda}((d)) \prod_{(i, j) \in \lambda} \frac{1-q t^{j-i}}{1-e^{\epsilon} t^{j-i}}=\frac{1}{\epsilon} \delta_{1, \kappa(\lambda)} \frac{1-t^{d}}{d} \frac{s_{\lambda}(\mathbf{p}(q, t))}{s_{\lambda}\left(\mathbf{p}_{\infty}\right)}+O(1)
$$

where we use the notations of Lemma 圆 and where $O(1)$ denotes terms of order $\epsilon^{k}, k=0,1, \ldots$

The Lemma follows from (47), from $s_{\lambda}\left(\mathbf{p}_{\infty}\right)=\frac{\operatorname{dim} \lambda}{d !}$, and from the formula (7) where power sums are specified by (48), in particular we have

$$
s_{\lambda}\left(\mathbf{p}\left(e^{\epsilon}, \mathrm{t}\right)\right)=\epsilon \frac{\operatorname{dim} \lambda}{d !} \varphi_{\lambda}((d)) \frac{d}{1-\mathrm{t}^{d}}+o(\epsilon)
$$

(C) The third one is based on choosing $t$ to be close to the root of unity $t_{0}=e^{\frac{2 \pi}{d} \sqrt{-1}}$, and is as follows. Let $\lambda$ be a one-hook partition: $\kappa(\lambda)=1$. As we know $\varphi_{\lambda}((d)) \neq 0$ in this case, see Lemma 2 It is interesting to see what is going on with (477) in case $\mathrm{t}=e^{\frac{2 \pi}{d} \sqrt{-1}} e^{\epsilon}$, where $\epsilon$ is small, and $\mathrm{q}=\mathrm{t}^{K}$ with $K$ being a positive integer, and $K<d$. In this case $w\left(\Delta, \mathrm{t}_{0}^{K}, \mathrm{t}_{0}\right)$ is a finite number which a polynomial in $\mathrm{t}_{0}$. Let $\tilde{\mathrm{q}}$ be fixed and $\tilde{\mathrm{q}}^{d} \neq 1$. Then

$$
\varphi_{\lambda}((d)) \prod_{(i, j) \in \lambda} \frac{1-\mathrm{t}^{K+j-i}}{1-\tilde{\mathrm{q}} \mathrm{t}^{j-i}}=\varphi_{\lambda}((d)) \frac{s_{\lambda}\left(\mathbf{p}\left(\mathrm{t}^{K}, \mathrm{t}\right)\right)}{s_{\lambda}(\mathbf{p}(\tilde{\mathrm{q}}, \mathrm{t}))}=\epsilon \frac{d}{1-\tilde{\mathrm{q}}^{d}} \frac{s_{\lambda}\left(\mathbf{p}\left(\mathrm{t}_{0}^{K}, \mathrm{t}_{0}\right)\right)}{s_{\lambda}\left(\mathbf{p}_{\infty}\right)} \delta_{1, \kappa(\lambda)}+o(\epsilon)
$$


We also get

$$
T_{\lambda}\left(\mathrm{t}_{0} e^{\epsilon}\right)=\epsilon \frac{d^{2}}{\varphi_{\lambda}((d))}+o(\epsilon), \quad s_{\lambda}\left(\mathbf{p}\left(0, \mathrm{t}_{0} e^{\epsilon}\right)\right)=\frac{1}{\epsilon d} \varphi_{\lambda}((d))+O(1)
$$

Let us note the similarity of relations (48)-(49) to the scalar product of the power sums symmetric functions where the Macdonald's symmetric functions are orthogonal, see [40]. We have

Remark 15. For $\xi_{0}=\xi_{-}=0$. Let us re-write eq. (51) as follows

$$
\prod_{(i . j) \in \lambda} r(\xi, x+j-i \mid \mathrm{t})=e^{\sum_{m>0} \frac{1}{m}\left(1-\mathrm{t}^{m}\right) \mathrm{p}_{m}^{*} \mathrm{t}^{m x-m} T_{\lambda}\left(\mathrm{t}^{m}\right)}=\sum_{\mu} \mathrm{t}^{(x-1)|\lambda|} P_{\mu}\left(\mathrm{p}^{*} ; 0, \mathrm{t}\right) Q_{\mu}\left(\mathrm{T}_{\lambda, \mathrm{t}} ; 0, \mathrm{t}\right)
$$

where $P_{\mu}$ and $Q_{\mu}$ are Macdonald polynomials with parameters $\mathrm{q}$ and $\mathrm{t}$ evaluated at the $\mathrm{q}=0$ (namely, these are Hall-Littlewood polynomials). Here the notations are the same as in [40, however here $P_{\mu}$ and $Q_{\mu}$ are written as functions of power sums variables which are $\mathrm{p}^{*}=\left(\mathrm{p}_{1}^{*}, \mathrm{p}_{2}^{*}, \mathrm{p}_{3}^{*}, \ldots\right)$, for $P_{\mu}$ and $\mathrm{T}_{\lambda, \mathrm{t}}=\left(T_{\lambda}(\mathrm{t}), T_{\lambda}\left(\mathrm{t}^{2}\right), T_{\lambda}\left(\mathrm{t}^{3}\right), \ldots\right)$ for the second Hall-Littlewood polynomial $Q_{\mu}$. Polynomials $Q_{\mu}$ may be also viewed as the symmetric functions of $d$ quantum contents $\mathrm{t}^{j-i},(i, j) \in \lambda$. We remind [40] that the scalar products of power sums and of the Macdonald polynomials with the parameters $q$ and $t$ may be written as

$$
<p_{\lambda}, p_{\mu}>=z_{\mu} \prod_{i=1}^{\ell(\mu)} \frac{1-\mathrm{q}^{\mu_{i}}}{1-\mathrm{t}^{\mu_{i}}} \delta_{\mu, \lambda}, \quad<P_{\lambda}, Q_{\mu}>=\delta_{\mu, \lambda}
$$

The number $z_{\mu}$ is defined by (12) below. The origin of the appearance of the Hall-Littlewood polynomials is not clear.

\section{Weighted sums of Hurwitz numbers}

Below we will consider combinations of normalized characters written as follows

$$
\sum_{\substack{|\lambda|=d \\ \ell(\lambda) \leq N}}(*) \varphi_{\lambda}(\Delta) \frac{\operatorname{dim} \lambda}{d !}
$$

where $(*)$ denotes a chosen (polynomial or not polynomial) function in many variables where the role of variables play the normalized characters $\varphi_{\lambda}$ evaluated at all possible different partitions of the number $d$. According to (1) in case $d \leq N$ this sum is a weighted sum of the projective Hurwitz numbers. However the parameter $N$ is an arbitrary integer and may be chosen large enough, so in this work we will not care about this unequality. The point that the sums below may obtained as specifications of $\mathrm{H}_{r}(d, \Delta)$ of (8) resulting from the choice of $r$ in either (4) or (5). Different examples of specifications presented also in Section 6 .

Weighted sums below may be compared to the weighted sums studied in [28, 24] where statistics of the $\mathbb{C P}^{1}$ Hurwitz numbers compatible with the property of the integrability of the related generating series was studied. Let us notice that though we can not choose functions $\left(^{*}\right)$ in an arbitrary way, there are infinitely many ways to choose them, we are interested in those which are related to BKP tau functions in a natural way.

The factor $\left(^{*}\right)$ appears due to the content product in the formula for hypergeometric tau functions.

Weighted sums below are labeled by a given partition $\mu=\left(\mu_{1}, \mu_{2}, \ldots\right)$. Our examples are as follows.

\subsection{Parametrization I}

In case (4) we will weight Hurwitz with the help of symmetric functions of contents viewed as functions of the power sums variable, the role of power sums play $\left(\Phi_{1}(\lambda), \Phi_{2}(\lambda), \ldots\right)$ defined by (32) and $\left(\phi_{1}(\lambda), \phi_{2}(\lambda), \ldots\right)$ defined by (26) .

(a) Hurwitz numbers weighted by power sums monomials built of $\left(\Phi_{1}(\lambda), \Phi_{2}(\lambda), \ldots\right), \Phi_{\mu}(\lambda):=$ $\prod_{i=1}^{\ell(\mu)} \Phi_{\mu_{i}}(\lambda)$ :

$$
\mathrm{C}_{\mu}(d ; \Delta):=\sum_{\substack{\lambda \\|\lambda|=d}} \Phi_{\mu}(\lambda) \varphi_{\lambda}(\Delta) \frac{\operatorname{dim} \lambda}{d !}
$$


This is a linear combination of Hurwitz numbers of (both connected and disconnected) $d$-fold covers with the profile $\Delta$ at $\infty$ and $\ell(\mu)$ different branch points, and Euler characteristic of the covers is $\mathrm{E}^{\prime}=\ell(\Delta)-d-|\mu|$. This follows from the Hurwitz formula for a $d$-fold covering $\mathrm{E}^{\prime}-E d=\sum_{i}\left(\ell\left(\Delta_{i}\right)-d\right)$ where the sum range over all branch points, where $\mathrm{E}^{\prime}$ and $\mathrm{E}$ are Euler characteristic respectively of the cover and of the base.

In case we choose $\mu=\left(1^{b}\right)$, the integer $\mathrm{C}_{\mu}(d ; \Delta)$ counts the number of branched non-equivalent coverings of the projective plane with a given ramification profile at some point and $b$ simple branch points

$$
\mathrm{C}_{\left(1^{b}\right)}(\Delta)=H_{\mathbb{R P}^{2}}(d ; \underbrace{\Gamma, \ldots, \Gamma}_{b}, \Delta), \quad|\Gamma|=|\Delta|=d
$$

For $\mu=\left(1^{b} 2\right)$ by 35 we obtain

$$
\mathrm{C}_{\left(1^{b} 2\right)}(\Delta)=-H_{\mathbb{R}^{2}}(d ; \underbrace{\Gamma, \ldots, \Gamma}_{b+2}, \Delta)+2 H_{\mathbb{R}^{2}}(d ; \underbrace{\Gamma, \ldots, \Gamma}_{b},\left(1^{d-4} 2^{2}\right), \Delta)+2 H_{\mathbb{R}^{2}}(d ; \underbrace{\Gamma, \ldots, \Gamma}_{b},\left(1^{d-3} 3^{1}\right), \Delta)
$$

(b) Hurwitz numbers weighted by Jack polynomials. In our case the Jack polynomials are homogeneous symmetric polynomials in $d$ variables which are integers - contents of all nodes of $\lambda$. At the same time the Jack polynomials may be re-written as the (quasihomogeneous) polynomials in power sum variables - in the integers $\left(\Phi_{1}(\lambda), \Phi_{2}(\lambda), \ldots\right.$ ) (which in turn are also quasihomogeneous in the variables $\phi_{k}(\lambda)$ according to (32) $)$. The last fact allows to use the content product to define the weighted Hurwitz numbers as follows:

$$
\mathrm{J}_{\mu}^{(\alpha)}(d ; \Delta):=\sum_{\substack{\lambda \\|\lambda|=d}} Q_{\mu}^{(\alpha)}(\Phi(\lambda)) \varphi_{\lambda}(\Delta) \frac{\operatorname{dim} \lambda}{d !}
$$

where $Q_{\lambda}^{(\alpha)}$ is the (dual) Jack polynomial in notations of Ch VI, sec 10 of [40].

The Euler characteristic of the cover is $\mathrm{E}^{\prime}=\ell(\Delta)-d-|\mu|$ similar to the previous example.

(c) Perhaps the most important example is the sum of Hurwitz numbers which may be called projective Goulden-Jackson Hurwitz number (compare to [19])

$$
\mathrm{S}_{\mu}(d ; \Delta):=\sum_{\lambda} \frac{\operatorname{dim} \lambda}{d !} \varphi_{\lambda}(\Delta) \prod_{s=1}^{k} \phi_{\mu_{s}}(\lambda)=\sum_{\substack{\Delta^{1}, \ldots, \Delta^{s} \\ \ell^{*}\left(\Delta^{s}\right)=\mu_{s}, s=1, \ldots, k}} H_{\mathbb{R}^{2}}\left(d ; \Delta^{1}, \ldots, \Delta^{k}, \Delta\right)
$$

which is the sum of the Hurwitz numbers of all $d$-branched covers of $\mathbb{R P}^{2}$ with $k+1$ ramification profiles given by an arbitrary partition $\Delta$ and partitions $\Delta^{s}, s=1, \ldots, k$ whose lengths are given numbers: $\ell\left(\Delta^{s}\right)=d-\mu_{s}$. The Euler characteristic of the cover is $\mathrm{E}^{\prime}=\ell(\Delta)-d-|\mu|$. In (60) $\phi_{\mu}(\lambda):=\prod_{i=1}^{\ell(\mu)} \phi_{\mu_{i}}(\lambda)$ where $\phi_{i}$ were introduced in (26). Actually each weighted sum of Hurwitz numbers obtained from BKP is a linear combination of (60).

Remark 16. Let us select the case where the sum $S_{\mu}$ reduces to a single term, thus it is not the sum of Hurwitz numbers but a Hurwitz number itself. We occurs if we choose the partition $\mu$ to be $\mu(b, m):=\left(1^{b}(d-1)^{m}\right)$. We get

$$
\mathrm{S}_{\mu(b, m)}(d, \Delta)=H^{1, b+m+1}(d ; \Delta, \underbrace{\Gamma, \ldots, \Gamma}_{b}, \underbrace{(d), \ldots,(d)}_{m})
$$

which counts $d$-fold covers of $\mathbb{R P}^{2}$ with the following set if ramification profiles: an arbitrary profile $\Delta$, say, over 0 , and $b$ simple branch points and also $m$ maximally ramified profiles. In case $m>0$ this Hurwitz number coincides with the Hurwitz numbers of connected covers. It results from Remarks 35 .

\subsection{Parametrisation II}

In case (4) we will weight Hurwitz with the help of symmetric functions viewed as functions of the power sums variable, the role of the set of power sums play the set $\left(T_{\lambda}(\mathrm{t}), T_{\lambda}\left(\mathrm{t}^{2}\right), \ldots\right)$ defined by (43). We denote this set by $\mathrm{T}_{\lambda, t}$. In this case weighted sums contain Hurwitz numbers for covers with different Euler characteristic therefore there is no sense to introduce the analogue of the constant $h$.

In the examples below the prefactor $\left(^{*}\right)$ is not a polynomial function of $\varphi_{\lambda}$. For a given partition $\mu$ we introduce t-dependent sums 
(d) Hurwitz numbers weighted by the power sums monomials built of $\left(T_{\lambda}(\mathrm{t}), T_{\lambda}\left(\mathrm{t}^{2}\right), \ldots\right)$

$$
\mathrm{K}_{\mu}(d ; \Delta \mid \mathrm{t}):=\sum_{\substack{\lambda \\|\lambda|=d}} T_{\lambda}(\mu \mid \mathrm{t}) \varphi_{\lambda}(\Delta) \frac{\operatorname{dim} \lambda}{d !}, \quad|\Delta|=d
$$

where $T_{\lambda}(\mu \mid \mathrm{t})=\prod_{i=1}^{\ell(\mu)} T_{\lambda}\left(\mathrm{t}^{\mu_{i}}\right)$ and $T_{\lambda}\left(\mathrm{t}^{\mu_{i}}\right)$ are defined by (43).

(e) t-dependent sums weighted by Jack polynomials

$$
\mathrm{J}_{\mu}^{(\alpha)}(d ; \Delta \mid \mathrm{t}):=\sum_{\substack{\lambda \\|\lambda|=d}} Q_{\mu}^{(\alpha)}\left(\mathrm{T}_{\lambda, \mathrm{t}}\right) \varphi_{\lambda}(\Delta) \frac{\operatorname{dim} \lambda}{d !}, \quad|\Delta|=d
$$

where $Q_{\mu}^{(\alpha)}$ is the Jack polynomial. It may be viewed either as the homogeneous symmetric function of the $d$ variables - quantum contents of the diagram $\lambda$, or, alternatively, as the quasihomogeneous functions of power sums variables $\mathrm{T}_{\lambda, \mathrm{t}}=\left(T_{\lambda}(\mathrm{t}), T_{\lambda}\left(\mathrm{t}^{2}\right), T_{\lambda}\left(\mathrm{t}^{3}\right), \ldots\right)$ expressed in terms of $S_{d}$ characters via Lemma 4 (see also Remark 15).

(f) sums weighted by Macdonald polynomials

$$
\mathrm{M}_{\mu}^{\mathrm{q}, \mathrm{t}}(d ; \Delta):=\sum_{\substack{\lambda \\|\lambda|=d}} Q_{\mu}^{\mathrm{q}, \mathrm{t}}\left(\mathrm{T}_{\lambda, \mathrm{t}}\right) \varphi_{\lambda}(\Delta) \frac{\operatorname{dim} \lambda}{d !}, \quad|\Delta|=d
$$

where $Q_{\mu}^{\mathrm{q}, \mathrm{t}}\left(\mathrm{T}_{\lambda, \mathrm{t}}\right)$ are Macdonald polynomials which are viewed as functions of power sums variables which are $\mathrm{T}_{\lambda, \mathrm{t}}=\left(T_{\lambda}(\mathrm{t}), T_{\lambda}\left(\mathrm{t}^{2}\right), T_{\lambda}\left(\mathrm{t}^{3}\right), \ldots\right)$ (see Remark 15). Here the polynomials $Q_{\mu}^{\mathrm{q}, \mathrm{t}}$ may be also written as symmetric functions in $d$ variables which are the quantum contents of the diagram $\lambda$.

Let us note that the idea to weight (the $\mathbb{C P}^{1}$ ) Hurwitz numbers by symmetric functions first was worked out in [24] where $\left\{h_{\mu}, m_{\mu}\right\},\left\{e_{\mu}, f_{\mu}\right\}$ also $\left\{s_{\mu}\right\}$ and $\left\{p_{\mu}\right\}$ basis sets (see [40]) were used. The notion of $q$-deformed Hurwitz numbers introduced in 24] in our approach is based on $q$-dependent specifications of the parameters $\zeta$ in the parametrization I (4), the parametrization II (5) was not considered in [24].

(g) Remark 14 suggests to consider the following weighted sums of Hurwitz numbers

$$
\begin{gathered}
\mathrm{F}\left(d, \Delta,(d),\left\{\mathrm{q}_{s}, \mathrm{t}_{s}\right\}\right):=\sum_{\substack{\lambda \\
|\lambda|=d}} \varphi_{\lambda}((d)) \varphi_{\lambda}(\Delta) \frac{\operatorname{dim} \lambda}{d !} \prod_{s=1}^{k} \frac{s_{\lambda}\left(\mathbf{p}\left(\mathrm{q}_{s}, \mathrm{t}_{s}\right)\right)}{s_{\lambda}\left(\mathbf{p}_{\infty}\right)} \\
=\sum_{\substack{\lambda \\
|\lambda|=d}} \varphi_{\lambda}((d)) \varphi_{\lambda}(\Delta) \frac{\operatorname{dim} \lambda}{d !} \prod_{s=1}^{k}\left(1+\sum_{\mu \neq 1^{d}} \varphi_{\lambda}(\mu) w\left(\Delta^{(s)}, \mathrm{q}_{s}, \mathrm{t}_{s}\right)\right)
\end{gathered}
$$

As we see this sum describes covers with the following set of profiles on $\mathbb{R P}^{2}$ : an arbitrary profile $\Delta$ over 0 , the maximally ramified profile $(d)$ over another point, special weighted sums of profiles $\Delta^{(s)}, s=1, \ldots, k$ over each of $k$ additional branch points with the ramification weights $w\left(\Delta^{(s)}, \mathrm{q}_{s}, \mathrm{t}_{s}\right)$ of (49). (Here we skip details because it will published in a more detailed text. Such sums allow to count the $d$-fold covers whose profiles $\Delta^{(s)}$ over the additional branch points contain given numbers of parts which are multiples of other given numbers playing the role of a chosen set of degrees of roots of unity. This is achieved by studying limits where the parameters $\mathrm{q}_{s}$ and $\mathrm{t}_{s}$ are chosen to be close to the roots of unity.)

We will show that the numbers $\mathrm{C}_{\mu}(d ; \Delta), \mathrm{J}_{\mu}(d ; \Delta), \mathrm{S}(d ; \Delta)$ and $\mathrm{K}_{\mu}(d ; \Delta \mid \mathrm{t}), \mathrm{M}_{\mu}^{\mathrm{q}, \mathrm{t}}(d ; \Delta), \mathrm{F}\left(d, \Delta,(d),\left\{\mathrm{q}_{s}, \mathrm{t}_{s}\right\}\right)$ are generated by special BKP tau functions considered in Sections 6 and 7 . For instance the number (60) is generated by (83) and the number (65) is generated by (95) below.

\section{BKP tau functions.}

\subsection{BKP hierarchy of Kac and van de Leur.}

There are two different BKP hierarchies of integrable equations, one was introduced by the Kyoto group in 31, the other was introduced by V. Kac and J. van de Leur in 32. We need the last one. This hierarchy includes the celebrated KP one as a particular reduction. In a certain way (see [39]) the BKP 
hierarchy may be related to the three-component KP hierarchy introduced in 31 (earlier described in 61 with the help of L-A pairs of differential operators with matrix valued coefficients). For a detailed description of the BKP we refer readers to the original work [32], and here we write down the first non-trivial equations (Hirota equations) for the BKP tau function. These are

$$
\begin{aligned}
& \frac{1}{2} \frac{\partial \tau(N, n, \mathbf{p})}{\partial p_{2}} \tau(N+1, n+1, \mathbf{p})-\frac{1}{2} \tau(N, n, \mathbf{p}) \frac{\partial \tau(N+1, n+1, \mathbf{p})}{\partial p_{2}}+\frac{1}{2} \frac{\partial^{2} \tau(N, n, \mathbf{p})}{\partial^{2} p_{1}} \tau(N+1, n+1, \mathbf{p}) \\
&+\frac{1}{2} \tau(N, n, \mathbf{p}) \frac{\partial^{2} \tau(N+1, n+1, \mathbf{p})}{\partial^{2} p_{1}}-\frac{\partial \tau(N, n, \mathbf{p})}{\partial p_{1}} \frac{\partial \tau(N+1, n+1, \mathbf{p})}{\partial p_{1}}=\tau(N+2, n+2, \mathbf{p}) \tau(N-1, n-1, \mathbf{p})(66)
\end{aligned}
$$

The BKP tau functions depend on the set of higher times $t_{m}=\frac{1}{m} p_{m}, m>0$ and the discrete parameter $N$. In [58] a second discrete parameter $n$ was added, and the simplest Hirota equation relating the BKP tau functions for neighboring values of $n$ is

$$
\begin{array}{r}
\frac{1}{2} \tau(N, n+1, \mathbf{p}) \frac{\partial^{2} \tau(N+1, n+1, \mathbf{p})}{\partial^{2} p_{1}}-\frac{1}{2} \frac{\tau(N, n+1, \mathbf{p})}{\partial^{2} p_{1}} \tau(N+1, n+1, \mathbf{p})= \\
\frac{\partial \tau(N+2, n+2, \mathbf{p})}{\partial p_{1}} \tau(N-1, n, \mathbf{p})-\frac{\partial \tau(N+1, n+2, \mathbf{p})}{\partial p_{1}} \tau(N, n, \mathbf{p})
\end{array}
$$

The complete set of Hirota equations with two discrete parameters is written down in the Appendix. The general solution to the BKP Hirota equations may be written as

$$
\tau(N, n, \mathbf{p})=\sum_{\lambda} A_{\lambda}(N, n) s_{\lambda}(\mathbf{p})
$$

where $A_{\lambda}$ satisfies the Plucker relations for an isotropic Grassmannian and (as one can show with the help of the Wick formula) may be written in pfaffian form.

\subsection{BKP tau function of the hypergeometric type.}

We are interested in a certain subclass of the BKP tau functions (68) introduced in [58] and called BKP hypergeometric tau functions, which may be compared to a similar class of TL and KP tau functions found in [36, 55.

Similarly to [55] we proceed as follows. Suppose that $\lambda$ is a Young diagram. Given an arbitrary function $r$ of one variable we construct the following product

$$
r_{\lambda}(x):=\prod_{(i . j) \in \lambda} r(x+j-i)
$$

which is called the content product (or, sometimes, the generalized Pochhammer symbol attached to a Young diagram $\lambda$ ). Examples were considered above.

Remark 17. (1) If $r=f g$, then $r_{\lambda}(x)=f_{\lambda}(x) g_{\lambda}(x)$. (2) If $\tilde{r}(x)=(r(x))^{n}, n \in \mathbb{C}$, then $\tilde{r}_{\lambda}(x)=\left(r_{\lambda}(x)\right)^{n}$.

We consider sums over partitions of the form

$$
\sum_{\substack{\lambda \\ \ell(\lambda) \leq N}} r_{\lambda}(n) c^{|\lambda|} s_{\lambda}(\mathbf{p})=: \tau_{r}^{\mathrm{B}}(N, n, \mathbf{p})
$$

where $s_{\lambda}$ are the Schur functions [40] and $\mathbf{p}$ denotes the semi-infinite set $\left(p_{1}, p_{2}, \ldots\right)$. It was shown in [58] that up to a factor (70) defines the BKP tau function:

Proposition 4. Any given $r$ the tau function $g(n) \tau_{r}^{\mathrm{B}}(N, n, \mathbf{p})$ solves the BKP Hirota equations. Here $g(n)$ is a function of the parameter $n$ defined by (120) in the Appendix B.

Let us mark two points: though discrete parameters enter Hirota equations, for our purposes (a) the factor $g(n)$ is unimportant (b) the cutting $N$ should be chosen large enough and we can take $N=+\infty$.

We call such tau functions hypergeometric because both the so-called generalized hypergeometric functions and the basic hypergeometric functions of one variable may be obtained as specifications of 
(170). For instance one can choose $p_{m}=x^{m}$. Then a rational function $r$ in (70) yields the generalized hypergeometric function while trigonometric $r$ results in the basic one. However the key tau function is the simplest one:

Example. Consider $r(x)=1$ for any $x$. Such tau function does not depend on $n$ and will be denoted by $\tau_{1}(N, \mathbf{p})$. Other hypergeometric tau functions may be obtained by action of a specially chosen vertex operator on $\tau_{1}(N, \mathbf{p})$, for example see (75). If we take $N=+\infty$ we can obtain

$$
\tau_{1}^{\mathrm{B}}(\infty, \mathbf{p})=\sum_{\lambda} c^{|\lambda|} s_{\lambda}(\mathbf{p})=e^{\sum_{m>0}\left(\frac{c^{2}}{2 m} p_{m}^{2}+c \frac{p_{2 m-1}}{2 m-1}\right)}
$$

Remark 18. Each tau function $\tau_{r}^{\mathrm{B}}$ may be expressed as a pfaffian, see [58].

2KP and BKP hypergeometric tau functions. The role of the hypergeometric functions of matrix argument in form of KP tau functions presented in [57] was marked in [19] in the context of combinatorial problems. Hypergeometric tau function of the two-component KP $(2 \mathrm{KP})$ may be written as

$$
\sum_{\substack{\lambda \\ \ell(\lambda) \leq N}} r_{\lambda}(n) c^{|\lambda|} s_{\lambda}(\mathbf{p}) s_{\lambda}(\overline{\mathbf{p}})=: \tau_{r}^{2 \mathrm{KP}}(N, n, \mathbf{p}, \overline{\mathbf{p}})
$$

where $r_{\lambda}(n)$ is the same as in (70). Here two independent sets $\mathbf{p}=\left(p_{1}, p_{2}, \ldots\right)$ and $\overline{\mathbf{p}}=\left(\bar{p}_{1}, \bar{p}_{2},\right)$ and two discrete parameters $N$ and $n$ play the role of $2 \mathrm{KP}$ higher times. (We do not mark the dependence of the right hand side on the constant $c$ since it is trivial.) Then hypergeometric tau functions of $2 \mathrm{KP}$ and BKP hierarchies are related:

$$
\left[e^{\sum_{i>0} \frac{i}{2} \frac{\partial^{2}}{\partial \bar{p}_{i}^{2}}+\sum_{i>0, \text { odd } \frac{\partial}{\partial \bar{p}_{i}}}} \cdot \tau_{r}^{2 \mathrm{KP}}(N, n, \mathbf{p}, \overline{\mathbf{p}})\right]_{\overline{\mathbf{p}}=0}=\tau_{r}^{\mathrm{B}}(N, n, \mathbf{p})
$$

which follows from (18) and (19):

$$
\left[e^{\sum_{i>0} \frac{i}{2} \frac{\partial^{2}}{\partial p_{i}^{2}}+\sum_{i>0, \text { odd }} \frac{\partial}{\partial p_{i}}} \cdot s_{\lambda}(\mathbf{p})\right]_{\mathbf{p}=0}=1
$$

Hypergeometric tau functions via the vertex operators. From bosonization formulae of 31 in [58, tau functions (3) were presented in terms of an action of the vertex operators. For $r$ given by (5) (the same: by (50)) the tau function (3) may be written as

$$
\tau_{r}^{B}(N, n, \mathbf{p})=\frac{1}{g(n)} e^{\xi_{0} \hat{h}_{2}(n) \log \mathrm{t}+\sum_{m \neq 0} \mathrm{p}_{m}^{*} \hat{h}\left(n, \mathrm{t}^{m}\right)} \cdot \sum_{\substack{\lambda \\ \ell(\lambda) \leq N}} c^{|\lambda|} s_{\lambda}(\mathbf{p})
$$

where $\hat{h}\left(n, \mathrm{t}^{m}\right)(m \in \mathbb{Z})$ are commuting operators defined as vertex operators

$$
\hat{h}(n, \mathrm{t}):=\mathrm{t}^{n} \operatorname{res}_{z} \frac{d z}{z} e^{\sum_{i>0}\left(\mathrm{t}^{i}-1\right) \frac{z^{i} p_{i}}{i}} e^{-\sum_{i>0}\left(\mathrm{t}^{-i}-1\right) z^{-i} \frac{\partial}{\partial p_{i}}}
$$

and where $\hat{h}_{2}(n)$ is determined by the generating series $\hat{h}\left(n, e^{\epsilon}\right)=: 1+\sum_{i \geq 0} \frac{\epsilon^{i+1}}{(i+1) !} \hat{h}_{i}(n)$. The operators $\hat{h}_{i}(n)$ were written down in [1] in the most explicit way. From (76) we get

$$
\begin{gathered}
\hat{h}_{0}(n)=n, \quad \hat{h}_{1}(n)=n^{2}+\sum_{i>0} i p_{i} \frac{\partial}{\partial p_{i}} \\
\hat{h}_{2}(n)=n^{3}+\sum_{i, j}\left((i+j) p_{i} p_{j} \frac{\partial}{\partial p_{i+j}}+i j p_{i+j} \frac{\partial^{2}}{\partial p_{i} \partial p_{j}}\right)
\end{gathered}
$$

In particular the operator $\hat{h}_{2}(0)$ is known as the cut-and-join operator which was introduced in [20]. 
For $r$ given by (4) (the same: by (38)) tau function (3) may be written as

$$
\tau_{r}^{B}(N, n, \mathbf{p})=\frac{1}{g(n)} e^{\sum_{m>0} p_{m}^{*} \hat{h}_{m}(n)} \cdot \sum_{\lambda \ell(\lambda) \leq N} c^{|\lambda|} s_{\lambda}(\mathbf{p})
$$

Example. For $N=+\infty, n=0$ and $r(x)=e^{\zeta_{1} x}$ (that is $p_{m}^{*}=0, m>2$, see (41)) we get

$$
\tau_{r}^{\mathrm{B}}(\mathbf{p})=\sum_{\lambda} e^{\zeta_{1} \varphi_{\lambda}(\Gamma)} c^{|\lambda|} s_{\lambda}(\mathbf{p})=e^{\zeta_{1} \hat{h}_{2}(0)} \cdot e^{\sum_{m>0} \frac{c^{2}}{2 m} p_{m}^{2}+\frac{c}{2 m-1} p_{2 m-1}}
$$

\section{Examples of the BKP hypergeometric tau functions.}

In case we use parameters to describe $r$, say, the parameters $\zeta$ as in (4), we shall write $\tau^{\mathrm{B}}(N, n, \mathbf{p} \mid \zeta)$ instead of $\tau_{r}^{B}(N, n, \mathbf{p})$. Let us use Propositions 20 and relations (36), (47) to construct examples of BKP tau functions. In view of (8) each example may be considered as the generating function for certain sums of Hurwitz numbers. More specified examples are the subject of Section 7 where tau functions generating sums introduced in Section 4 .

Example 0. The simplest hypergeometric tau function is $\sum_{\ell(\lambda) \leq N} s_{\lambda}(\mathbf{p})$ is related to $\zeta=0$.

Example I. First we choose (38) for the content product. Using (17) we write down the following example

$$
\tau^{\mathrm{B}}(N, 0, \mathbf{p} \mid h, \zeta)=\sum_{d \geq 0} c^{d} \sum_{\substack{\lambda \\|\lambda|=d, \ell(\lambda) \leq N}} s_{\lambda}(\mathbf{p}) \exp \sum_{m>0} \frac{1}{m} h^{m} \zeta_{m} \Phi_{m}(\lambda)
$$

If $h=1$ it may be suitable to introduce the dependence on the variable $n$ after performing the triangle change of variables $\zeta \rightarrow \mathbf{p}^{*}$ given by $V(x-1, \zeta)-V(x, \zeta)=V\left(x, \mathbf{p}^{*}\right)$. Then

$$
\tau^{\mathrm{B}}\left(N, n, \mathbf{p} \mid \mathbf{p}^{*}\right)=\sum_{d \geq 0} c^{d} \sum_{\substack{\lambda \\|\lambda|=d, \ell(\lambda) \leq N}} s_{\lambda}(\mathbf{p}) \prod_{i=1}^{N} e^{V\left(h_{i+n}(\lambda), \mathbf{p}^{*}\right)}
$$

where $h_{i}(\lambda)=\lambda_{i}-i$.

Remark 19. The specialization $p_{m}=\operatorname{tr} R^{m}=\sum_{a=1}^{N} x_{a}^{m}$, where $x_{i}=e^{y_{i}}$, allows (79) to be rewritten as

$$
\tau^{\mathrm{B}}(N, 0, \mathbf{p} \mid \zeta)=\frac{1}{\Delta_{N}(\mathbf{x})} \sum_{h_{1}, \ldots, h_{N}=1}^{M} e^{V\left(h, \mathbf{p}^{*}\right)} \operatorname{det}\left(e^{y_{j} h_{i}}\right) \operatorname{sgn} \Delta_{N}(h)
$$

which is a discrete analogue of the two-matrix integral

$$
\int d U \int d R \operatorname{det} R^{n} \exp \left(\operatorname{Tr}\left(U Y U^{\dagger} R+\sum_{m \neq 0} \frac{1}{m} p_{m}^{*} R^{m}\right)\right)
$$

where the first integral represents integration over unitary matrices and the second is the integral over real symmetric ones, $d U$ and $d R$ denote the corresponding Haar measures. $Y$ is any diagonal matrix (a source). The matrices are $N$ by $N$ ones. This integral may be viewed as an analogue of the Kontsevich integral.

Example Ia. In (79) one can specify the variables $\zeta$ as

$$
\zeta_{m}=\sum_{s=1}^{k} \mathrm{n}_{s}\left(-\mathrm{a}_{s}\right)^{-m}, \quad \zeta_{0}=-\mathrm{n}_{s} \log \mathrm{a}_{s}
$$

where $\mathrm{a}_{s} \in \mathbb{C}$. If we restore the dependence of tau function on $n$ we obtain

$$
\tau^{\mathrm{B}}\left(N, n, \mathbf{p} \mid h,\left\{\mathbf{a}_{s}, \mathrm{n}_{s}\right\}\right)=\sum_{d \geq 0} c^{d} \sum_{\substack{\lambda \\|\lambda|=d, \ell(\lambda) \leq N}} s_{\lambda}(\mathbf{p}) \prod_{s=1}^{k} \prod_{(i, j) \in \lambda}\left(1+h \frac{n+j-i}{a_{s}}\right)^{-\mathrm{n}_{s}}
$$


where $\mathrm{a}$ and $\mathrm{n}$ are respectively the collections of complex parameters $\mathrm{a}_{1}, \ldots, \mathrm{a}_{k}$ and $\mathrm{n}_{1}, \ldots, \mathrm{n}_{k}$. For $\mathrm{n}_{s}= \pm$ we obtain the pfaffian version of the hypergeometric function of matrix argument [57.

Example Ib. Let us take all $\mathrm{n}_{s}$ equal to $\mathrm{n}(\alpha)=\frac{1}{\alpha}$ in the previous example. We obtain

$$
\tau^{\mathrm{B}}(N, n, \mathbf{p} \mid h, \mathrm{a}, \mathrm{n}(\alpha))=\sum_{d \geq 0} c^{d} \sum_{\substack{\lambda \\|\lambda|=d, \ell(\lambda) \leq N}} s_{\lambda}(\mathbf{p}) \sum_{\mu} h^{|\mu|} P_{\mu}^{(\alpha)}(-\mathrm{a}(n)) Q_{\mu}^{(\alpha)}(\Phi(\lambda))
$$

where $P_{\mu}^{\alpha}$ and $Q_{\mu}^{\alpha}$ is the pair of dual Jack polynomials written in the notation of Ch IV [40]. Here the first Jack polynomial, $P_{\mu}^{\alpha}$, is a symmetric function of the variables $-\mathrm{a}(n)=\left(-a_{1}-n, \ldots,-a_{k}-n\right)$ while the second Jack polynomial $Q_{\mu}^{\alpha}(\Phi(\lambda))$ should be viewed as quasihomogeneous polynomial in power sum variables $\Phi=\left(\Phi_{1}(\lambda), \Phi_{2}(\lambda), \ldots\right)$.

Example II. Next we use (50) and (17) getting

$$
\begin{aligned}
\tau^{\mathrm{B}}\left(N, n, \mathbf{p} \mid\left\{\mathbf{p}^{*(s)}, \mathrm{t}_{s}\right\}\right)=\sum_{d \geq 0} c^{d} \sum_{\substack{\lambda \\
|\lambda|=d, \ell(\lambda) \leq N}} s_{\lambda}(\mathbf{p}) \prod_{s=1}^{k} e^{\xi_{0}\left(\varphi_{\lambda}(\Gamma)+n d\right) \log \mathrm{t}_{s}+\sum_{m \neq 0} \xi_{m}^{(s)} \mathrm{t}_{s}^{m n} T_{\lambda}\left(\mathrm{t}_{s}^{m}\right)} \\
=\sum_{d \geq 0} c^{d} \sum_{\substack{\lambda \\
|\lambda|=d, \ell(\lambda) \leq N}} s_{\lambda}(\mathbf{p}) \prod_{s=1}^{k} e^{\xi_{0}\left(\varphi_{\lambda}(\Gamma)+n d\right) \log \mathrm{t}_{s}-\sum_{m \neq 0} \frac{1}{m}\left(1-\mathrm{t}_{s}^{m}\right) \mathrm{p}_{m}^{*(s)} \mathrm{t}_{s}^{m x-m} T_{\lambda}\left(\mathrm{t}_{s}^{m}\right)}
\end{aligned}
$$

The variables $\mathrm{p}^{*(s)}$ are related to the variables $\xi^{(s)}$ by $\mathrm{p}_{m}^{*}=\xi_{m} \frac{\mathrm{t}^{m}}{\mathrm{t}^{m}-1}$.

For $k=1$ (here we will re-denote $\mathrm{p}^{*(1)} \rightarrow-\mathrm{p}^{*}$ ) and $\mathrm{p}_{m}^{*}=0, m<0$ we have

$$
\tau^{\mathrm{B}}\left(N, n, \mathbf{p} \mid \mathrm{p}^{*}, \mathbf{t}\right)=\sum_{d \geq 0} c^{d} \sum_{\substack{\lambda \\|\lambda|=d, \ell(\lambda) \leq N}} s_{\lambda}(\mathbf{p}) \prod_{s=1}^{k} \mathrm{t}^{\xi_{0} \varphi_{\lambda}(\Gamma)+d \xi_{0}} \sum_{\mu} P_{\mu}^{0, \mathrm{t}}\left(\mathrm{p}^{*}\right) Q_{\mu}^{0, \mathrm{t}}\left(T_{\lambda, \mathrm{t}}\right)
$$

where $P_{\lambda}^{0, \mathrm{t}}$ and $Q_{\lambda}^{0, \mathrm{t}}$ are the Macdonald polynomials specified by $\mathrm{q}=0$ (Hall-Littlewood polynomials) may be written either as the quasihomogeneous polynomial of the power sum variables $T_{\lambda, \mathrm{t}}=$ $\left(T_{\lambda}(\mathrm{t}), T_{\lambda}\left(\mathrm{t}^{2}\right), \ldots\right)$, or as the symmetric polynomial in quantum contents, see Remark 15.

Remark 20. Given $s$ let us specify $\mathbf{p}=\mathbf{p}(\mathbf{q}, \mathrm{t})$ according to (48). Then the series (85) solves the BKP Hirota equations with respect to the variables $\mathrm{p}^{*}$. In case $|\mathrm{t}|=1$ and is not a root of $1, \tau^{\mathrm{B}}$ of (85) is basically a discrete version of the circular $\beta=1$ ensemble

$$
\frac{1}{N !} \sum_{h_{1}, \ldots, h_{N}} \prod_{i<j}\left|\mathrm{t}^{h_{i}}-\mathrm{t}^{h_{j}}\right| \prod_{i=1}^{N} e^{V\left(\mathrm{p}^{*}, \mathrm{t}^{h_{i}}\right)} \mu\left(h_{1} ; \mathrm{q}, \mathrm{t}\right)
$$

with a certain weight function $\mu$ independent of $\mathrm{p}^{*}$, see [58. This may be compared to Remark 11 and to the discrete version of the orthogonal ensemble (10).

Consider three specifications of the variables $\xi$ in (85).

Example IIa. First, we put each $\xi_{m}^{(s)}=0, s=1, \ldots, k$. Then the content product depends only on the parameter $\xi_{0}$. We obtain the BKP analogue of the Okounkov's TL tau function presented in [51]:

$$
\tau^{\mathrm{B}}\left(N, n, \mathbf{p} \mid \xi_{0}\right)=\sum_{d \geq 0} c^{d} \sum_{\substack{\lambda \\|\lambda|=d, \ell(\lambda) \leq N}} s_{\lambda}(\mathbf{p}) \prod_{(i . j) \in \lambda} e^{(n+j-i) \xi_{0}}
$$

Example IIb. Now, take $\xi_{0}=0$ and

$$
\xi_{m}^{(s)}=\frac{\mathrm{t}^{m}-1}{\mathrm{t}^{m}} \mathrm{p}_{m}^{*(s)}=\mathrm{n}_{s} \mathrm{q}_{s}^{m}, \quad m>0
$$

We obtain

$$
\tau^{\mathrm{B}}\left(N, n, \mathbf{p} \mid\left\{\mathrm{t}_{s}, \mathrm{q}_{s}, \mathrm{n}_{s}\right\}\right)=\sum_{d \geq 0} c^{d} \sum_{\substack{\lambda \\|\lambda|=d, \ell(\lambda) \leq N}} s_{\lambda}(\mathbf{p}) \prod_{s=1}^{k} \prod_{(i . j) \in \lambda}\left(1-\mathrm{q}_{s} \mathrm{t}_{s}^{n+j-i}\right)^{-\mathrm{n}_{s}}
$$


where $\mathrm{t}, \mathrm{q}, \mathrm{n}$ are sets of complex numbers $\mathrm{t}_{s}, \mathrm{q}_{s}, \mathrm{n}_{s}, s=1, \ldots, k$.

In case $\mathrm{n}_{s}= \pm 1, s=1, \ldots, k$ the tau function (90) is the pfaffian version of Milne's hypergeometric function [56].

Example IIc. Next, take $\xi_{0}=0$ and

$$
\xi_{ \pm m}^{(s)}=\frac{\mathrm{t}^{ \pm m}-1}{\mathrm{t}^{ \pm m}} \mathbf{p}_{ \pm m}^{*(s)}=(-1)^{m} \mathrm{n}_{s} \frac{\mathrm{q}_{s}^{\frac{m}{2}} \mathbf{t}_{s}^{ \pm \mathbf{a}_{s} m}}{1-\mathbf{q}_{s}^{m}} \quad s=1, \ldots, k, \quad m>0
$$

and put $\mathrm{q}_{s}=e^{2 \pi i \tau_{s}}, \mathrm{t}_{s}=e^{2 c_{s} \pi i}$. Then (85) takes the form

$$
\tau^{\mathrm{B}}\left(N, n, \mathbf{p} \mid\left\{\mathbf{c}_{s}, \tau_{s}, \mathrm{a}_{s}, \mathbf{n}_{s}\right\}\right)=\sum_{d \geq 0} c^{d} \sum_{\substack{\lambda \\|\lambda|=d, \ell(\lambda) \leq N}} s_{\lambda}(\mathbf{p}) \prod_{s=1}^{k}\left(\theta_{\lambda}\left(\mathrm{c}_{s}\left(n+\mathrm{a}_{s}\right), \tau_{s}\right)\right)^{-\mathrm{n}_{s}}
$$

where $\{\mathrm{c}, \tau, \mathrm{a}, \mathrm{n}\}$ are sets of complex numbers $\left\{\mathrm{c}_{s}, \tau_{s}, \mathrm{a}_{s}, \mathrm{n}_{s}, s=1, \ldots, k\right\}$, and where

$$
\theta_{\lambda}\left(c_{s}\left(n+\mathrm{a}_{s}\right), \tau_{s}\right):=\prod_{(i . j) \in \lambda} \theta\left(c_{s}\left(n+\mathrm{a}_{s}+j-i\right), \tau_{s}\right)
$$

is the elliptic version of the Pochhammer symbol, $\theta$ is the Jacobi theta function

$$
\theta\left(c_{s} x, \tau_{s}\right):=\sum_{k \in \mathbb{Z}} \exp \left(\pi i k^{2} \tau_{s}+2 c_{s} \pi i k x\right)=\left(\mathrm{q}_{s} ; \mathrm{q}_{s}\right)_{\infty} \prod_{k=1}^{\infty}\left(1+\mathrm{q}_{s}^{k-\frac{1}{2}} \mathrm{t}_{s}^{x}\right)\left(1+\mathrm{q}_{s}^{k-\frac{1}{2}} \mathrm{t}_{s}^{-x}\right)
$$

where $\left(\mathrm{q}_{s} ; \mathrm{q}_{s}\right)_{\infty}$ is the Dedekind function. For this example we chose $c=\left(\mathrm{q}_{s} ; \mathrm{q}_{s}\right)_{\infty}$ in (85). For $\mathrm{n}_{s}= \pm 1$ we obtain the pfaffian version of an elliptic hypergeometric function considered in [55].

Example IId. In (87) we choose $k=1, \mathrm{n}=1$. Let us take take

$$
\xi_{m}=\frac{1-\mathrm{t}^{m}}{1-\mathrm{q}^{m}} \sum_{i=1}^{k} y_{i}^{m}, \quad m>0
$$

all other variables vanish. This may be viewed as a limiting case of the previous Example Ib where we send $k \rightarrow \infty$. Then

$$
r(x)=\prod_{m>0} \prod_{i=1}^{k} \frac{1-y_{i} q^{m} \mathrm{t}^{x+1}}{1-y_{i} q^{m} \mathrm{t}^{x}}
$$

The content product is equal to

$$
\prod_{(i . j) \in \lambda} \prod_{m>0} \prod_{i=s}^{k} \frac{1-y_{i} q^{m} \mathrm{t}^{x+1+j-i}}{1-y_{s} q^{m} \mathrm{t}^{x+j-i}}=e^{\sum_{m>0} \frac{1}{m} \frac{1-\mathrm{t}^{m}}{1-\mathrm{q}^{m} \mathrm{t}^{m x}} T_{\lambda}\left(\mathrm{t}^{m}\right) \sum_{i=1}^{k} y_{i}^{m}}=\sum_{\mu} \mathrm{t}^{x|\mu|} P_{\mu}^{\mathrm{q}, \mathrm{t}}(Y) Q_{\mu}^{\mathrm{q}, \mathrm{t}}\left(T_{\lambda, \mathrm{t}}\right)
$$

where the Macdonald function $P_{\mu}^{\mathrm{q}, \mathrm{t}}$ is the symmetric polynomial in $Y=\left(y_{1}, \ldots, y_{k}\right)$ and the Macdonald function $Q_{\mu}^{\mathrm{q}, \mathrm{t}}$ may be written either as the quasihomogeneous polynomial of the power sum variables $T_{\lambda, \mathrm{t}}=\left(T_{\lambda}(\mathrm{t}), T_{\lambda}\left(\mathrm{t}^{2}\right), \ldots\right)$, or as the symmetric polynomial in quantum contents, see Remark 15 The tau function (87) takes the form

$$
\begin{aligned}
\tau^{\mathrm{B}}\left(N, n, \mathbf{p} \mid \mathbf{q}, \mathrm{t}, \xi_{0}, Y\right) & =\sum_{d \geq 0} c^{d} \sum_{\substack{\lambda \\
|\lambda|=d, \ell(\lambda) \leq N}} s_{\lambda}(\mathbf{p}) e^{\xi_{0} \varphi_{\lambda}(\Gamma)} \sum_{\mu} \mathrm{t}^{n|\mu|} P_{\mu}^{\mathrm{q}, \mathrm{t}}(Y) Q_{\mu}^{\mathbf{q}, \mathrm{t}}\left(T_{\lambda, \mathrm{t}}\right) \\
& =\sum_{d \geq 0} c^{d} \sum_{\substack{\lambda \\
|\lambda|=d, \ell(\lambda) \leq N}} \prod_{j=1}^{N} e^{\xi_{0}\left(\lambda_{j}-j+n\right)^{2}} s_{\lambda}(\mathbf{p}) \prod_{j=1}^{N} \prod_{i=1}^{k} \prod_{m>0} e^{\frac{y_{i}^{m}}{1-q^{m} \mathrm{t}} \mathrm{t}^{m\left(\lambda_{j}-j+n-1\right.}}
\end{aligned}
$$

where $P_{\mu}^{\mathrm{q}, \mathrm{t}}$ and $Q_{\mu}^{\mathrm{q}, \mathrm{t}}$ are Macdonald polynomials, see Remark [15. The last equality follows from (52).

Example III. Let us choose

$$
r(x)=(a+x) \prod_{s=1}^{k} \frac{1-\mathrm{q}_{s} \mathrm{t}_{s}^{x}}{1-e^{\epsilon_{s}} \mathrm{t}_{s}}\left(a_{s}+x\right)
$$


where we used both parameterizations (see Remark [17). We obtain the following tau function

$$
\tau^{\mathrm{B}}\left(N, n, \mathbf{p} \mid a,\left\{a_{s}, \mathrm{t}_{s}, \epsilon_{s}\right\}\right)=\sum_{d \geq 0} c^{d} \sum_{\substack{\lambda \\|\lambda|=d}} s_{\lambda}(\mathbf{p}) \frac{s_{\lambda}(\mathbf{p}(a))}{s_{\lambda}\left(\mathbf{p}_{\infty}\right)} \prod_{s} \frac{s_{\lambda}\left(\mathbf{p}\left(\mathrm{q}_{s}, \mathrm{t}_{s}\right)\right)}{s_{\lambda}\left(\mathbf{p}\left(e^{\epsilon_{s}}, \mathrm{t}_{s}\right)\right)} \frac{s_{\lambda}\left(\mathbf{p}\left(a_{s}\right)\right)}{s_{\lambda}\left(\mathbf{p}_{\infty}\right)}
$$

In particular these tau functions generate sums $\mathrm{F}\left(d, \Delta,(d),\left\{\mathrm{q}_{s}, \mathrm{t}_{s}\right\}\right)$ of (65).

Remark 21. Formula (83) may be obtained as a limiting case of (90) if we take $\mathrm{q}_{s}=\mathrm{t}_{s}^{\mathrm{a}_{s}}$ and send $\mathrm{t} \rightarrow 1$ taking into account that for the hypergeometric tau functions (70) there is the obvious transformation $r_{\lambda} \rightarrow$ $a^{-|\lambda|} r_{\lambda}, p_{m} \rightarrow a p_{m}, m>0$, which leaves them unchanged.

In this limiting case polynomials $P^{\mathrm{q}, \mathrm{t}}$ and $Q^{\mathrm{q}, \mathrm{t}}$ goes to Jack polynomials [40, compare to (84).

Remark 22. Similarly to (73) one may prove the relation

$$
\left.e^{\frac{1}{2} \sum_{m>0} m \frac{\partial^{2}}{\partial p_{m}^{2}}+\sum_{m>0, \text { odd }} \frac{\partial}{\partial p_{m}}} \cdot \tau_{r}^{\mathrm{B}}(N, n, \mathbf{p})\right|_{\mathbf{p}=0}=\sum_{d \geq 0} c^{d} \sum_{\substack{\lambda \\|\lambda|=d, \ell(\lambda) \leq N}} \prod_{(i . j) \in \lambda} r(n+j-i)
$$

where the right hand side generates weighted Hurwitz numbers for the torus and the Klein bottle.

\section{BKP tau functions generating Hurwitz numbers}

\subsection{Hurwitz numbers themselves}

As we shall see the hypergeometric tau functions generate weighted sums of Hurwitz numbers. However there exist special cases when one gets Hurwitz numbers themselves, this is based on Remark 5 .

We will make difference between the parameterizations I and II.

First, let us write down the simplest case of a single branch point related to all $r=1$ and $N=\infty$. This case is generated by $\tau_{1}^{\mathrm{B}}$ where it is reasonable to produce the change $p_{m} \rightarrow h^{-1} c^{m} p_{m}$. We get

$$
e^{\frac{1}{h^{2}} \sum_{m>0} \frac{1}{2 m} p_{m}^{2} c^{2 m}+\frac{1}{h} \sum_{m o d d} \frac{1}{m} p_{m} c^{m}}=\sum_{d>0} c^{d} \sum_{\mathrm{E}^{\prime} \leq d} h^{-\mathrm{E}^{\prime}} \sum_{\substack{\Delta \\|\Delta|=d, \ell(\Delta)=\mathrm{E}^{\prime}}} \mathbf{p}_{\Delta} H^{1,1}(d ; \Delta)
$$

where $H^{1,1}(d ; \Delta)$ is the Hurwitz number describing $d$-fold covering of $\mathbb{R P}^{2}$ with a single branch point of type $\Delta=\left(d_{1}, \ldots, d_{l}\right),|\Delta|=d$ by a (not necessarily connected) Klein surface of Euler characteristic $\mathrm{E}^{\prime}=\ell(\Delta)$. For instance, for $d=3, \mathrm{E}^{\prime}=1$ we get $H^{1,1}(\Delta)=\frac{1}{3} \delta_{\Delta,(3)}$. In particular for unbranched covering we get formula (13).

Next let us notice that the exponent of the left hand side may be rewritten as the generating series of the connected Hurwitz numbers

$$
\frac{1}{h^{2}} \sum_{d=2 m} c^{2 m} p_{m}^{2} H_{\text {con }}^{1,1}(d ;(m, m))+\frac{1}{h} \sum_{d=2 m-1} c^{2 m-1} p_{2 m-1} H_{\text {con }}^{1,1}(d ;(2 m-1))
$$

where $H_{\text {con }}^{1,1}$ describes $d$-fold covering either by the Riemann sphere $(d=2 m)$ or by the projective plane $(d=2 m-1)$. These are the only ways to cover $\mathbb{R P}^{2}$ by a connected surface for the case of the single branch point. The geometrical meaning of the exponent in (97) may be explained as follows. The projective plain may be viewed as the unit disk with the identification of the opposite points $z$ and $-z$ on the boarder: $|z|=1$. In case we cover the Riemann sphere by the Riemann sphere $z \rightarrow z^{m}$ we get two critical points with the same profiles. However we cover $\mathbb{R P}^{2}$ by the Riemann sphere, then we have the composition of the mapping $z \rightarrow z^{m}$ on the Riemann sphere and the factorization by antipodal involution $z \rightarrow-\frac{1}{z}$. Thus we have the ramification profile $(m, m)$ at the single critical point 0 of $\mathbb{R P}^{2}$. The automorphism group is the dihedral group of the order $2 m$ which consists of rotations on $\frac{2 \pi}{m}$ and antipodal involution $z \rightarrow-\frac{1}{\bar{z}}$. Thus we get that $H_{\text {con }}^{1,1}(d ;(m, m))=\frac{1}{2 m}$ which is the factor in the first sum in the exponent in (97). Now let us cover $\mathbb{R P}^{2}$ by $\mathbb{R P}^{2}$ via $z \rightarrow z^{d}$. For even $d$ we have the critical point 0 , in addition each point of the unit circle $|z|=1$ is critical (a folding), while from the beginning we restrict our consideration only on isolated critical points. For odd $d=2 m-1$ there is the single critical point 0 , the automorphism group consists of rotations on the angle $\frac{2 \pi}{2 m-1}$. Thus in this case $H^{1,1}(d ;(2 m-1))=\frac{1}{2 m-1}$ which is the factor in the second sum in the exponent in (97). 
Next, let us consider BKP hypergeometric function in the parametrization I where we put $\zeta_{1}=$ $\beta+\sum_{i=1}^{L} a_{i}^{-1}$ and $\zeta_{k}=\sum_{i=1}^{L} a_{i}^{-k}$

$$
H^{1, b+c+1}(d ; \underbrace{\Gamma, \ldots, \Gamma}_{b}, \underbrace{(d), \ldots,(d)}_{m}, \Delta)=c^{-d} h^{\mathrm{E}^{\prime}}[\tau(N=+\infty, 0, \mathbf{p} \mid \zeta)]_{b, m}
$$

where the brackets $[*]_{b, m}$ means the picking up the factor of the term $\mathbf{p}_{\Delta} \prod_{i=1} a_{i}^{-1}$ which counts $d$-fold covers of $\mathbb{R P}^{2}$ with the following ramification type: there are $b$ simple branch points, $m$ branch points of type $(d)$ and one branch point of type $\Delta=\left(d_{1}, \ldots, d_{l}\right)$. Each cover is a connected Klein surface in case $m>0$ and not necessarily connected one in case $m=0$ )

of Euler characteristic $\mathrm{E}^{\prime}=d-b-m(d-1)$. See Proposition 1 on the properties of Hurwitz numbers regarding branch points with the profiles $(d)$ (maximally ramified branch points).

\subsection{BKP tau function as generating function for the weighted sums of Hur- witz numbers.}

In this subsection the power of $\frac{1}{h}$ counts the Euler characteristic of the covering surface denoted by $\mathrm{E}^{\prime}$.

For this purpose we change

$$
p_{m}=h^{-1} \tilde{p}_{m}
$$

First of all we present the simplest weighted sum of Hurwitz numbers which is just the sum of Hurwitz numbers related to a single branch point with a fixed Euler characteristic $\mathrm{E}^{\prime}$

$$
\sum_{d \geq 0} c^{d} \sum_{\ell} h^{d-l} \sum_{\substack{\Delta^{(i)} \\ \ell\left(\Delta^{(i)}\right)=l}} H^{1,1}\left(d ; \Delta^{(i)}\right)=\frac{1}{\left(1-c^{2}\right)^{h^{-2}}}\left(\frac{1+c}{1-c}\right)^{h^{-1}}
$$

where each $\Delta^{(i)}$ has the same weight $d$ and length $\ell$.

From previous sections we found

Proposition 5. The tau function (79) generates the numbers $C_{\mu}(\Delta)$ (57) through

$$
\tau^{\mathrm{B}}(N, 0, \mathbf{p} \mid h, \zeta)=\sum_{d \geq 0} c^{d} \sum_{\substack{\mu, \Delta \\|\Delta|=d}} h^{|\mu|-\ell(\Delta)} \frac{1}{z_{\mu}} C_{\mu}(\Delta) \zeta_{\mu} \mathbf{p}_{\Delta}
$$

where $z_{\mu}$ is defined by (12). For $d=|\Delta| \leq N$ the numbers $C_{\mu}(\Delta)$ are weighted Hurwitz numbers.

Corollary 1. In particular, let us put $\zeta_{m}=0$ if $m>1$. Then (101) reads

$$
\sum_{d \geq 0} c^{d} \sum_{\substack{\lambda \\|\lambda|=d, \ell(\lambda) \leq N}} e^{h \zeta_{1} \varphi_{\lambda}(\Gamma)} s_{\lambda}(\mathbf{p})=\sum_{d, b \geq 0} c^{d} \sum_{\substack{\Delta \\|\Delta|=d}} h^{b-\ell(\Delta)} \tilde{\mathbf{p}}_{\Delta} \frac{\zeta_{1}^{b}}{b !} H(d ; \underbrace{\Gamma, \ldots, \Gamma}_{b}, \Delta)
$$

which is the $\mathbb{R P}^{2}$ analogue of the Okounkov generating function [51].

The representation of this series in the form of a matrix integral is written down below, see (108).

Weighted sums of Hurwitz numbers generated by the BKP tau functions (90) and (83) were written down in our previous work [50]. The simplest example resulting from (83) is similar to one considered in [28, and may be presented as follows. The tau function (83), with $\mathrm{n}_{s}=1$ for $s=1, \ldots, k$, generates sums $S$ defined by (60):

Proposition 6. For the discussion compare to Example 2.22 in 34

$$
\begin{gathered}
\tau^{\mathrm{B}}\left(N, n, \mathbf{p} \mid h,\left\{a_{s}\right\}\right)=\sum_{d \geq 0} c^{d} \sum_{\substack{\lambda \\
|\lambda|=d, \ell(\lambda) \leq N}} s_{\lambda}(\mathbf{p}) \prod_{s=1}^{k} \prod_{(i . j) \in \lambda}\left(a_{s} h^{-1}+n+j-i\right) \\
=\sum_{d \geq 0} c^{d} \sum_{\substack{\Delta \\
|\Delta|=d}} \sum_{\mu} \frac{1}{d !}\left(a_{s} h^{-1}+n\right)^{d-\mu_{s}} \mathbf{p}_{\Delta} S_{\mu}(d, \Delta)
\end{gathered}
$$


Proposition 7. The tau function (85) generates the numbers $K_{\mu^{(s)}}\left(\Delta \mid t_{s}\right)$ (62) through

$$
\tau^{\mathrm{B}}\left(N, n, \mathbf{p} \mid \xi,\left\{t_{s}\right\}\right)=\sum_{d \geq 0} c^{d} \sum_{\substack{\mu, \Delta \\|\Delta|=d}} \prod_{s=1}^{k} \frac{1}{d ! z_{\mu}} \mathbf{p}_{\Delta} \xi_{\mu^{(s)}} K_{\mu^{(s)}}\left(\Delta \mid t_{s}\right)
$$

where $z_{\mu}$ is defined by (12). For $d=|\Delta| \leq N$ the numbers $K_{\mu^{(s)}}\left(\Delta \mid t_{s}\right)$ are weighted Hurwitz numbers.

Proposition 8. The tau function (93) generates the Hurwitz numbers $M_{\mu}^{q, t}$ weighted by Macdonald polynomials (see 64):

$$
\tau^{\mathrm{B}}(N, n, \mathbf{p} \mid q, t, 0, Y)=\sum_{d \geq 0} c^{d} \sum_{\substack{\Delta \\|\Delta|=d}} \frac{1}{d !} \mathbf{p}_{\Delta} \sum_{\mu} t^{n|\mu|} P_{\mu}^{q, t}(Y) M_{\mu}^{q, t}(d ; \Delta)
$$

Proposition 9. The numbers $F\left(d, \Delta,(d),\left\{q_{s}, t_{s}\right\}\right)$ given by (65) may be obtained as the following term in the tau function (65):

$$
\begin{aligned}
\tau^{\mathrm{B}}(N, n, \mathbf{p} \mid a & \left.,\left\{a_{s}, t_{s}, \epsilon_{s}\right\}\right)=\sum_{d \geq 0} c^{d} \sum_{\lambda} s_{\lambda}(\mathbf{p}) \frac{s_{\lambda}(\mathbf{p}(a))}{s_{\lambda}\left(\mathbf{p}_{\infty}\right)} \prod_{s=1}^{k} \frac{s_{\lambda}\left(\mathbf{p}\left(q_{s}, t_{s}\right)\right)}{s_{\lambda}\left(\mathbf{p}\left(e^{\epsilon_{s}}, t_{s}\right)\right)} \frac{s_{\lambda}\left(\mathbf{p}\left(a_{s}\right)\right)}{s_{\lambda}\left(\mathbf{p}_{\infty}\right)} \\
& =\sum_{d \geq 0} c^{d} \sum_{\substack{\Delta \\
|\Delta|=d}} \mathbf{p}_{\Delta}\left(F\left(d, \Delta,(d),\left\{q_{s}, t_{s}\right\}\right) a \prod_{s=1}^{k} \frac{a_{s}}{\epsilon_{s}}+\ldots\right)
\end{aligned}
$$

where dots means terms of different order in any of $\epsilon_{s}, a_{s}(s=1, \ldots, k)$ and $a$.

\section{Matrix integrals as generating functions of Hurwitz numbers}

In case the base surface is $\mathbb{C P}^{1}$ the set of examples of matrix integrals generating Hurwitz numbers were studied in works [10, 41, 2, 9, 35, 37, 63. One can show that the perturbation series in coupling constants of these integrals (Feynman graphs) may be related to TL (KP and two-component $\mathrm{KP}$ ) hypergeometric tau functions. It actually means that these series generate Hurwitz numbers with at most two arbitrary profiles while other ones are subjects of certain conditions since the origin of additional profiles is the content product factors in hypergeometric tau functions (72).

Here, very briefly, we will write down few generating series for the $\mathbb{R P}^{2}$ Hurwitz numbers. These series may be not tau functions themselves but may be presented as integrals of tau functions of matrix argument. (The matrix argument, which we denote by a capital letter, say $X$, means that the power sum variables $\mathbf{p}$ are specified as $p_{i}=\operatorname{tr} X^{i}, i>0$. Then instead of $s_{\lambda}(\mathbf{p}), \tau(\mathbf{p})$ we write $s_{\lambda}(X)$ and $\left.\tau(X)\right)$. If a matrix integral in examples below is a BKP tau function then it generates Hurwitz numbers with a single arbitrary profile and all other are subjects of restrictions identical to those in $\mathbb{C P}^{1}$ case mentioned above. In all examples $V$ is given by (11). We also recall that the limiting values of $\mathbf{p}(\mathbf{q}, \mathrm{t})$ given by (48) may be $\mathbf{p}(a)=(a, a, \ldots)$ and $\mathbf{p}_{\infty}=(1,0,0, \ldots)$. We also recall that numbers $H^{\mathrm{E}, \mathrm{F}}(d ; \ldots)$ are Hurwitz numbers only in case $d \leq N, N$ is the size of matrices.

For more details of the $\mathbb{R P}^{2}$ case see [50]. New development in [50] with respect to the consideration in 60] is the usage of products of matrices. Here we shall consider a few examples. All examples include the simplest BKP tau function, of matrix argument $X$, [58, defined by (compare to [20)

$$
\tau_{1}^{\mathrm{B}}(X):=\sum_{\lambda} s_{\lambda}(X)=e^{\frac{1}{2} \sum_{m>0} \frac{1}{m}\left(\operatorname{tr} X^{m}\right)^{2}+\sum_{m>0, \text { odd }} \frac{1}{m} \operatorname{tr} X^{m}}=\frac{\operatorname{det}^{\frac{1}{2}} \frac{1+X}{1-X}}{\operatorname{det}^{\frac{1}{2}}\left(I_{N} \otimes I_{N}-X \otimes X\right)}
$$

as the part of the integration measure. Other integrands are the simplest $\mathrm{KP}$ tau functions $\tau_{1}^{\mathrm{KP}}(X, \mathbf{p}):=$ $e^{\operatorname{tr} V(X, \mathbf{p})}$ where $V$ is defined by (11) where the parameters $\mathbf{p}$ may be called coupling constants. The perturbation series in coupling constants are expressed as sums of products of the Schur functions over partitions and are similar to the series we considered in the previous sections. 
Example 1. The $\mathbb{R P}^{2}$ Okounkov Hurwitz series as a model of normal matrices. From the equality

$$
\left(2 \pi \zeta_{1}^{-1}\right)^{\frac{1}{2}} e^{\frac{\left(n \zeta_{0}\right)^{2}}{2 \zeta_{1}}} e^{\zeta_{0} n c+\frac{1}{2} \zeta_{1} c^{2}}=\int_{\mathbb{R}} e^{x_{i} n \zeta_{0}+\left(c x_{i}-\frac{1}{2} x_{i}^{2}\right) \zeta_{1}} d x_{i}
$$

in a similar way as was done in [59] using $\varphi_{\lambda}(\Gamma)=\sum_{(i . j) \in \lambda}(j-i)$, one can derive

$$
e^{n|\lambda| \zeta_{0}} e^{\zeta_{1} \varphi_{\lambda}(\Gamma)} \delta_{\lambda, \mu}=\mathrm{K} \int s_{\lambda}(M) s_{\mu}\left(M^{\dagger}\right) \operatorname{det}\left(M M^{\dagger}\right)^{n \zeta_{0}} e^{-\frac{1}{2} \zeta_{1} \operatorname{tr}\left(\log \left(M M^{\dagger}\right)\right)^{2}} d M
$$

where $\mathrm{K}$ is unimportant multiplier, $M$ is a normal matrix with eigenvalues $z_{1}, \ldots, z_{N}$ and $\log \left|z_{i}\right|=x_{i}$, and $d M=d_{*} U \prod_{i<j}\left|z_{i}-z_{j}\right|^{2} \prod_{i=1}^{N} d^{2} z_{i}$. Then the $\mathbb{R P}^{2}$ analogue of the Okounkov series (102) may be written

$$
\sum_{\substack{\lambda \\ \ell(\lambda) \leq N}} e^{n|\lambda| \zeta_{0}+\zeta_{1} \varphi_{\lambda}(\Gamma)} s_{\lambda}(\mathbf{p})=\mathrm{K} \int e^{V(M, \mathbf{p})} e^{\zeta_{0} n \operatorname{tr} \log \left(M M^{\dagger}\right)-\frac{1}{2} \zeta_{1}\left(\operatorname{tr} \log \left(M M^{\dagger}\right)\right)^{2}} \tau_{1}^{\mathrm{B}}\left(M^{\dagger}\right) d M
$$

A similar representation of the Okounkov $\mathbb{C P}^{1}$ series was earlier presented in 4 .

Below we use the following notations

- $d_{*} U$ is the normalized Haar measure on $\mathbb{U}(N): \int_{\mathbb{U}(N)} d_{*} U=1$

- $Z$ is a complex matrix

$$
d \Omega\left(Z, Z^{\dagger}\right)=\pi^{-n^{2}} e^{-\operatorname{tr}\left(Z Z^{\dagger}\right)} \prod_{i, j=1}^{N} d \Re Z_{i j} d \Im Z_{i j}
$$

- Let $M$ be a Hermitian matrix the measure is defined

$$
d M=\prod_{i \leq j} d \Re M_{i j} \prod_{i<j} d \Im M
$$

It is known 40

$$
\int s_{\lambda}(Z) s_{\mu}\left(Z^{\dagger}\right) d \Omega\left(Z, Z^{\dagger}\right)=(N)_{\lambda} \delta_{\lambda, \mu}
$$

where $(N)_{\lambda}:=\prod_{(i, j) \in \lambda}(N+j-i)$ is the Pochhammer symbol related to $\lambda$. A similar relation was used in [53, 27, 60, 2], 59, for models of Hermitian, complex and normal matrices.

By $I_{N}$ we shall denote the $N \times N$ unit matrix. We recall that

$$
s_{\lambda}\left(I_{N}\right)=(N)_{\lambda} s_{\lambda}\left(\mathbf{p}_{\infty}\right), \quad s_{\lambda}\left(\mathbf{p}_{\infty}\right)=\frac{\operatorname{dim} \lambda}{d !}, \quad d=|\lambda|
$$

Example 2. Three branch points. The generating function for $\mathbb{R P}^{2}$ Hurwitz numbers with three ramification points, having two arbitrary profiles at 0 and at $\infty$ with fixed length in the third point:

$$
\begin{gathered}
\sum_{\lambda} \frac{s_{\lambda}\left(I_{N}\right) s_{\lambda}\left(\mathbf{p}^{(1)}\right) s_{\lambda}\left(\mathbf{p}^{(2)}\right)}{\left(s_{\lambda}\left(\mathbf{p}_{\infty}\right)\right)^{2}} \\
=\int \tau_{1}^{\mathrm{B}}\left(Z_{1} Z_{2}\right) \prod_{i=1,2} e^{V\left(\operatorname{tr} Z_{i}^{\dagger}, \mathbf{p}^{(i)}\right)} d \Omega\left(Z_{i}, Z_{i}^{\dagger}\right)
\end{gathered}
$$

If $\mathbf{p}^{(2)}=\mathbf{p}(\mathbf{q}, \mathrm{t})$ with any given parameters $\mathbf{q}, \mathrm{t}$, then (110) is the hypergeometric BKP tau function. The same series over partitions may obtained from integrals where $Z, Z^{\dagger}$ are replaced either by normal matrices $M, M^{\dagger}$ or by a pair of Hermitian matrices (for these replacements see [50]).

Example 3. Hermitian two-matrix model. The following 'projective analogue' of the well-known two matrix model is the BKP tau function

$$
\int \tau_{1}^{\mathrm{B}}\left(c M_{2}\right) e^{\operatorname{tr} V\left(M_{1}, \mathbf{p}\right)+\operatorname{tr}\left(M_{1} M_{2}\right)} d M_{1} d M_{2}=\sum_{\lambda} c^{|\lambda|}(N)_{\lambda} s_{\lambda}(\mathbf{p})
$$


where $M_{1}, M_{2}$ are Hermitian matrices. Using results of [20] we can show that it is a projective analogue of the generating function of the so-called strictly monotonic Hurwitz numbers introduced by Goulden and Jackson. In the projective case these numbers counts paths on Cayley graph of the symmetric group whose initial point is a given partition while the end point is not fixed: we take the weighted sum over all possible end points, say, $\Delta$, the weight is given by $\chi(\Delta)$ of Lemma 1 .

Example 4. Unitary matrices. Generating series for projective Hurwitz numbers with arbitrary profiles in $n$ branch points and restricted profiles in other points:

$$
\begin{gathered}
\int e^{\operatorname{tr}\left(c U_{1}^{\dagger} \ldots U_{n+m}^{\dagger}\right)}\left(\prod_{i=n+1}^{n+m} \tau_{1}^{\mathrm{B}}\left(U_{i}\right) d_{*} U_{i}\right)\left(\prod_{i=1}^{n} \tau_{1}^{\mathrm{KP}}\left(U_{i}, \mathbf{p}^{(i)}\right) d_{*} U_{i}\right)= \\
\sum_{d \geq 0} c^{d}(d !)^{1-m} \sum_{\substack{\lambda,|\lambda|=d \\
\ell(\lambda) \leq N}}\left(\frac{\operatorname{dim} \lambda}{d !}\right)^{2-m}\left(\frac{s_{\lambda}\left(I_{N}\right)}{\operatorname{dim} \lambda}\right)^{1-m-n} \prod_{i=1}^{n} \frac{s_{\lambda}\left(\mathbf{p}^{(i)}\right)}{\operatorname{dim} \lambda}
\end{gathered}
$$

Here $\mathbf{p}^{(i)}$ are parameters. This series generate certain linear combination of Hurwitz numbers for base surfaces with Euler characteristic $2-m, m \geq 0$. The integral (111) is a BKP tau function in case the parameters are specialized as $\mathbf{p}^{(i)}=\mathbf{p}\left(\mathrm{q}_{i}, \mathrm{t}_{i}\right), i=2, \ldots, n$ with any values of $\mathrm{q}_{i}, \mathrm{t}_{i}$, and if in addition $m=1$. In case $n=1$ this BKP tau function may be viewed as an analogue of the generating function of the so-called non-connected Bousquet-Melou-Schaeffer numbers (see Example 2.16 in [34]). In case $n=m=1$ we obtain the following BKP tau function

$$
\int \tau_{1}^{\mathrm{B}}\left(U_{2}\right) e^{\operatorname{tr} V\left(U_{1}, \mathbf{p}\right)+\operatorname{tr}\left(c U_{1}^{\dagger} U_{2}^{\dagger}\right)} d_{*} U_{1} d_{*} U_{2}=\sum_{\substack{\lambda \\ \ell(\lambda) \leq N}} c^{|\lambda|} \frac{s_{\lambda}(\mathbf{p})}{(N)_{\lambda}}
$$

If we compare this series with ones used in [22, 23] we can see that it is a projective analogue of the generating function of the so-called weakly monotonic Hurwitz numbers. In the projective case it counts paths on Cayley graph whose initial point is a given partition while instead of a fixed end point we consider the sum over the all possible end points $\Delta$, with a weight given by $\chi(\Delta)$ of Lemma 1 .

Example 5. Integrals over complex matrices. A pair of examples. An analogue of Belyi curves generating function [63, [10] is as follows (compare also to (60)):

$$
\begin{gathered}
\sum_{\substack{\Delta^{(1)}, \ldots, \Delta^{(k+1)} \\
\ell\left(\Delta^{k+1}\right)=l}} \frac{N^{l}}{d !} H^{1, k+1}\left(d ; \Delta^{(1)}, \ldots, \Delta^{(k+1)}\right) \prod_{i=1}^{k+1} \mathbf{p}_{\Delta^{(i)}}^{(i)}=\sum_{\lambda}(N)_{\lambda} s_{\lambda}\left(\mathbf{p}^{(k+1)}\right) \prod_{i=1}^{k} \frac{s_{\lambda}\left(\mathbf{p}^{(i)}\right)}{s_{\lambda}\left(\mathbf{p}_{\infty}\right)} \\
=\int \tau_{1}^{\mathrm{KP}}\left(Z^{\dagger} Z_{1}^{\dagger} \cdots Z_{k}^{\dagger}, \mathbf{p}^{(k+1)}\right) \tau_{1}^{\mathrm{B}}(Z) d \Omega\left(Z, Z^{\dagger}\right) \prod_{i=1}^{k} \tau_{1}^{\mathrm{KP}}\left(Z_{i}, \mathbf{p}^{(i)}\right) d \Omega\left(Z_{i}, Z_{i}^{\dagger}\right)
\end{gathered}
$$

The series in the following example generates the projective Hurwitz numbers themselves where to get rid of the factor $(N)_{\lambda}$ in the sum over partitions we use mixed integration over $\mathbb{U}(N)$ and over complex matrices:

$$
\begin{gathered}
\sum_{\Delta^{(1)}, \ldots, \Delta(k+1)} \frac{c^{d}}{d !} H^{1, k+1}\left(d ; \Delta^{(1)}, \ldots, \Delta^{(k+1)}\right) \prod_{i=1}^{k+1} \mathbf{p}_{\Delta^{(i)}}^{(i)}=\sum_{\lambda, \ell(\lambda) \leq N} c^{|\lambda|} s_{\lambda}\left(\mathbf{p}^{(k+1)}\right) \prod_{i=1}^{k} \frac{s_{\lambda}\left(\mathbf{p}^{(i)}\right)}{s_{\lambda}\left(\mathbf{p}_{\infty}\right)} \\
=\int \tau_{1}^{\mathrm{KP}}\left(c U^{\dagger} Z_{1}^{\dagger} \cdots Z_{k}^{\dagger}, \mathbf{p}^{(k+1)}\right) \tau_{1}^{\mathrm{B}}(U) d_{*} U \prod_{i=1}^{k} \tau_{1}^{\mathrm{KP}}\left(Z_{i}, \mathbf{p}^{(i)}\right) d \Omega\left(Z_{i}, Z_{i}^{\dagger}\right)
\end{gathered}
$$

Here $Z, Z_{i}, i=1, \ldots, k$ are complex $N \times N$ matrices and $U \in \mathbb{U}(N)$. As in the previous examples one can specify all sets $\mathbf{p}^{(i)}=\mathbf{p}\left(\mathrm{q}_{i}, \mathrm{t}_{i}\right), i=1, \ldots, k+1$ except a single one which in this case has the meaning of the BKP higher times. 


\section{Acknowledgements}

A.O. was partially supported by RFBR grant 14-01-00860 and by V.E.Zakharov's scientific school (Program for Support of Leading Scientific Schools, grant NS-3753.2014.2) and also by by the Russian Academic Excellence Project '5-100'. The work of S.N. was partially supported by RFBR grants 15-5250041. We thank first of all J. Harnad, A. Mironov and J. van de Leur for important remarks, and also A. Zabrodin, S. Loktev and I. Marshall for useful discussions. Our special grates to L. Chekhov for the organization of the workshop on Hurwitz numbers (Moscow, May 2014) which inspired us to do this work.

\section{References}

[1] A. Alexandrov, A. Mironov, A. Morozov and S. Natanzon, "Integrability of Hurwitz Partition Functions. I. Summary", J.Phys.A: Math.Theor.45(2012) 045209, arXiv: 1103.4100

[2] A. Alexandrov, A. Mironov, A. Morozov and S. Natanzon, "On KP-integrable Hurwitz functions",JHEP 11(2014) 080, arXiv: 1405.1395

[3] A. Alexandrov, G. Chapuy, B. Eynard, J. Harnad, "Weighted Hurwitz numbers and topological recursion: an overview", arXiv:1610.09408, J. Harnad, Janosch Ortmann, "Semiclassical asymptotics of quantum weighted Hurwitz numbers", arXiv:1610.06280

[4] A. Alexandrov and A. V. Zabrodin "Free fermions and tau-functions", J.Geom.Phys. 67 (2013) pp. $37-80$; arXiv:1212.6049

[5] A. A. Alexeevski and S. M. Natanzon, "Noncommutative two-dimansional field theories and Hurwitz numbers for real algebraic curves", Selecta Math. N.S. v.12 (2006) ,n.3, pp. 307-377, arXiv:math/0202164

[6] A. V. Alekseevskii and S. M. Natanzon, "The algebra of bipartite graphs and Hurwitz numbers of seamed surfaces", Izvestiya Mathematics 72:4 (2008) pp. 627-646

[7] N. L. Alling and N.Greenleaf, "Fondation of the theory of Klein surfaces", Springer-Verlang, 1971, Leture Notes in Math. v. 219

[8] M. Aganagic, H. Ooguri, N. Saulina and C. Vafa, "Black holes, $q$-deformed 2D Yang-Mills and nonperturbative topological strings ", Nucl Phys B 715 (2005) pp. 304-348; R. Szabo and M.Tierz, "Chern-Simons matrix models, two-dimensional Yang-Mills theory, and the Surtherland model", arxiv preprint hep-th/1003.1228

[9] J. Ambjorn and L. Chekhov "The matrix model for hypergeometric Hurwitz number", arXiv:1409.3553

[10] J. Ambjorn and L. Chekhov, "The matrix model for dessins d'enfants", arXiv:1404.4240

[11] S. Bloch and A. Okounkov, "The Character of the Infinite Wedge Representation", arxiv:97120009

[12] S. R. Carrel, "The non-orientable map asymptotic constant $p_{g}$ ", arXiv:1406.1760

[13] R. Dijkgraaf, "Mirror symmetry and elliptic curves, The Moduli Space of Curves", R. Dijkgraaf, C. Faber, G. van der Geer (editors), Progress in Mathematics, 129, Birkhauser, 1995.

[14] P. Dunin-Barkowski, M. Kazarian, N. Orantin, S. Shadrin and L. Spitz, "Polynomiality of Hurwitz numbers, Bouchard-Marino conjecture, and a new proof of the ELSV formula", arXiv:1307.4729

[15] T. Ekedahl, S. K. Lando, V. Shapiro and A. Vainshtein, "On Hurwitz numbers and Hodge integrals", C.R. Acad. Sci. Paris Ser. I. Math. Vol. 146, N2, pp. 1175-1180 (1999)

[16] G. Frobenius, "Uber Gruppencharaktere", Sitzber, Kolniglich Preuss. Akad.Wiss.Berlin, (1896), pp. 985-1021 
[17] G. Frobenius and I. Schur, "Uber die reellen Darstellungen der endichen Druppen", Sitzber, Kolniglich Preuss. Akad.Wiss.Berlin, (1906), p. 186-208

[18] Gareth A. Jones, "Enumeration of Homomorphisms and Surface-Coverings", Quart. J. Math. Oxford (2), 46 (1995), pp. 485-507

[19] I. P. Goulden and D. M. Jackson, "The KP hierarchy, branched covers, and triangulations", Advances in Mathematics, 219 pp. 932-951, 2008

[20] I. P. Goulden and D. M. Jackson, "Transitive factorizations into transpositions and holomorphic mappings on the sphere", Proc. Amer. Math. Soc. 125(1) pp. 51-60 (1997).

[21] I. P. Goulden, M. Guay-Paquet and J. Novak ,"Monotone Hurwitz numbers and HCIZ integral", Ann. Math. Blaise Pascal 21 pp. 71-99 (2014)

[22] I. P. Goulden, M. Guay-Paquet, and J. Novak, "Monotone Hurwitz numbers in genus zero", Canad. J. Math. 65:5 (2013) pp. 1020-1042; arxiv: 1204.2618

[23] M. Guay-Paquet and J. Harnad, "2D Toda tau-functions as combinatorial generating functions", Letters in Mathematical Physics 105, pp. 827-852 (2015)

[24] M. Guay-Paquet and J. Harnad "Generating functions for weighted Hurwitz numbers", to appear in J. Math. Phys; arXiv:1408.6766

[25] J. Harnad and A. Yu. Orlov, "Scalar product of symmetric functions and matrix integrals", Theoretical and mathematical physics 137 (3), pp. 1676-1690 (2003)

[26] J. Harnad and A. Yu. Orlov, "Matrix integrals as Borel sums of Schur function expansions", Symmetry and Perturbation Theory 2002, Cala Gonoone (Sardinia), May 1-26, pp. (2002). Proceedings, pp. 116-123 (World Scientific, Singapore, eds. S. Abenda, G. Gaeta); arXiv:nlin/0209035

[27] J. Harnad and A. Yu. Orlov, "Fermionic construction of partition functions for two matrix models and perturbative Schur functions expansions", J. Phys. A 39, pp. 8783-8809 (2006)

[28] J. Harnad and A. Yu. Orlov, "Hypergeometric $\tau$-functions, Hurwitz numbers and enumeration of paths", Commun. Math. Phys. 338 (2015) pp. 267-284 arxiv: math.ph/1407.7800

[29] J. Harnad, "Multispecies quantum Hurwitz numbers", SIGMA 11, 097 (2015); arXiv:1410.8817

[30] J. Harnad, "Weighted Hurwitz numbers and hypergeometric $\tau$-functions: an overview", AMS Proc. Symp. Pure Math. 93 (2016) pp. 289-333 ; arXiv:1504.03408

[31] M. Jimbo and T. Miwa, "Solitons and Infinite Dimensional Lie Algebras", Publ. RIMS Kyoto Univ. 19, pp. 943-1001 (1983).

[32] V. Kac and J. van de Leur, "The Geometry of Spinors and the Multicomponent BKP and DKP Hierarchies", CRM Proceedings and Lecture Notes 14 (1998) pp. 159-202

[33] M. Kazarian and S. Lando, "Combinatorial solutions to integrable hierarchies", Uspekhi Mat. Nauk 70 (2015), no. 3(423), pp. 77-106. English translation: 2015 Russ. Math. Surv. 70, pp. 453-482; arXiv:1512.07172

[34] M. E. Kazarian and S. K. Lando, "An algebro-geometric proof of Witten's conjecture", J. Amer. Math. Soc. 20:4 (2007), pp. 1079-1089

[35] M. Kazarian and P. Zograph, "Virasoro constraints and topological recursion for Grothendieck's dessin counting", arxiv1406.5976

[36] S. Kharchev, A. Marshakov, A. Mironov and A. Morozov, "Generalized Kazakov-Migdal-Kontsevich Model: group theory aspects", International Journal of Mod Phys A10 (1995) p.2015

[37] S. K. Lando, A. K. Zvonkin Graphs on Surfaces and their Applications, Encyclopaedia of Mathematical Sciences, Volume 141, with appendix by D. Zagier, Springer, N.Y. (2004). 
[38] J. W. van de Leur, "Matrix Integrals and Geometry of Spinors", J. of Nonlinear Math. Phys. 8, pp. 288-311 (2001)

[39] J. W. van de Leur and A. Yu. Orlov, "Pfaffian and determinantal tau functions I ", Letters in Mathematical Physics 105 (11), pp. 1499-1531

[40] I.G. Macdonald, Symmetric Functions and Hall Polynomials, Clarendon Press, Oxford, (1995).

[41] R. de Mello Koch and S. Ramgoolam, "From Matrix Models and quantum fields to Hurwitz space and the absolute Galois group", arXiv: 1002.1634

[42] A. D. Mednykh, "Determination of the number of nonequivalent covering over a compact Riemann surface", Soviet Math. Dokl., 19(1978), pp. 318-320

[43] A. D. Mednykh and G. G. Pozdnyakova,"The number of nonequivalent covering over a compact nonorientable surface", Sibirs. Mat. Zh, 27(1986), +- 1, pp. 123-131,199

[44] A. D. Mironov, A. Yu. Morozov and S. M. Natanzon, "Complect set of cut-and-join operators in the Hurwitz-Kontsevich theory", Theor. and Math.Phys. 166:1,(2011), pp.1-22

[45] A. D. Mironov, A. Yu. Morozov and S. M. Natanzon, "Algebra of differential operators associated with Young diagramms", J.Geom.and Phys. n.62(2012), pp. 148-155

[46] M. Mulase and A. Waldron "Duality of Orthogonal and Symplectic Matrix Integrals and Quaternionic Feynman Graphs", arxiv:0206.011; E. Brezin and S. Hikami, "Intersection numbers from the antisymmetric Gaussian matrix model", arXiv:0804.4531

[47] S. M. Natanzon, "Klein surfaces", Russian Math.Surv., 45:6(1990), pp. 53-108.

[48] S. M. Natanzon, "Moduli of Riemann surfaces, real algebraic curves and their superanalogs", Translations of Math. Monograph, AMS, Vol.225 (2004), 160 p.

[49] S. M. Natanzon, "Simple Hurwitz numbers of a disk", Funk. Analysis ant its applications, v.44 (2010), n1, pp. 44-58

[50] S. M. Natanzon and A. Yu. Orlov, "Hurwitz numbers and BKP hierarchy", arXiv:1407.832

[51] A. Okounkov, "Toda equations for Hurwitz numbers", Math. Res. Lett. 7, pp. 447-453 (2000). See also arxivmath-004128.

[52] A. Okounkov and R. Pandharipande, "Gromov-Witten theory, Hurwitz theory and completed cycles", Annals of Math 163 p.517 (2006); arxiv math.AG/0204305

[53] A. Yu. Orlov, "Soliton theory, symmetric functions and matrix integrals", Acta Applicandae Mathematica 86 (1-2), pp. 131-158 (2005)

[54] A. Yu. Orlov, "Deformed Ginibre ensembles and integrable systems", Physics Letters A 378, pp. 319-328 (2014)

[55] A. Yu. Orlov and D. Scherbin, "Fermionic representation for basic hypergeometric functions related to Schur polynomials", arXiv preprint nlin/0001001

[56] A. Yu. Orlov and D. Scherbin, "Milne's hypergeometric functions in terms of free fermions", Journal of Physics A: Mathematical and General 34 (11), p.2295; S. C. Milne, "Summation theorems for basic hypergeometric series of Schur function argument", in Progress in Approximation Theory, Eds. A. A. Gonchar and E. B. Saff, pp. 51-77, Springer-Verlag, New-York, 1992

[57] A. Yu. Orlov and D. Scherbin, "Hypergeometric solutions of soliton equations", Theoretical and Mathematical Physics 128 (1), pp. 906-926 (2001)

[58] A. Yu. Orlov, T. Shiota and K. Takasaki, "Pfaffian structures and certain solutions to BKP hierarchies I. Sums over partitions", arXiv: math-ph/12014518; A. Yu. Orlov, T. Shiota and K. Takasaki, "Pfaffian structures and certain solutions to BKP hierarchies II. Multiple integrals", arxiv: nlin/1611. 
[59] A. Yu. Orlov and T. Shiota, "Schur function expansion for normal matrix model and associated discrete matrix models", Physics Letters A 343 (5), pp. 384-396

[60] A. Yu. Orlov, "New solvable matrix integrals" , Intern. J. Mod. Phys. A 19 (suppl 02), pp. 276-93 (2004).

[61] V. E. Zakharov and A. B. Shabat, J. Funct. Anal. Appl. 8, 226 (1974), 13, p.166 (1979)

[62] J. Zhou, "Hodge integrals, Hurwitz numbers and symmetric groups", arxiv preprint math/0308.024

[63] P. Zograf, "Enumeration of Gronthendieck's dessons and KP hierarchy", arXiv:1312.2538 (2013).

\section{A Hirota equations for the BKP tau function with two discrete time variables.}

The BKP hierarchy we are interested in was introduced in 32. In this paper the BKP tau function $\tau^{\mathrm{B}}(N, \mathbf{p})$ does not contain the discrete variable $n$. We need in a slightly general version of BKP hierarchy which includes $n$ as the higher time parameter, see [58 and 39. Hirota equations for the tau functions $\tau^{\mathrm{B}}(N, n, \mathbf{p})$ of this modified BKP hierarchy read

$$
\begin{array}{r}
\oint \frac{d z}{2 \pi i} z^{N^{\prime}-N-1} e^{V\left(\mathbf{p}^{\prime}-\mathbf{p}, z\right)} \tau\left(N^{\prime}-1, n, \mathbf{p}^{\prime}-\left[z^{-1}\right]\right) \tau\left(N+1, n+1, \mathbf{p}+\left[z^{-1}\right]\right) \\
+\oint \frac{d z}{2 \pi i} z^{N-N^{\prime}-3} e^{V\left(\mathbf{p}-\mathbf{p}^{\prime}, z\right)} \tau\left(N^{\prime}+1, n+2, \mathbf{p}^{\prime}+\left[z^{-1}\right]\right) \tau\left(N-1, n-1, \mathbf{p}-\left[z^{-1}\right]\right) \\
=\tau\left(N^{\prime}+1, n+1, \mathbf{p}^{\prime}\right) \tau(N-1, n, \mathbf{p})-\frac{1}{2}\left(1-(-1)^{N^{\prime}+N}\right) \tau\left(N^{\prime}, n+1, \mathbf{p}^{\prime} \mid g\right) \tau(N, n, \mathbf{p})
\end{array}
$$

and

$$
\begin{array}{r}
\oint \frac{d z}{2 \pi i} z^{N^{\prime}-N-2} e^{V\left(\mathbf{p}^{\prime}-\mathbf{p}, z\right)} \tau\left(N^{\prime}-1, n-1, \mathbf{p}^{\prime}-\left[z^{-1}\right]\right) \tau\left(N+1, n+1, \mathbf{p}+\left[z^{-1}\right]\right) \\
+\oint \frac{d z}{2 \pi i} z^{N-N^{\prime}-2} e^{V\left(\mathbf{p}-\mathbf{p}^{\prime}, z\right)} \tau\left(N^{\prime}+1, n+1, \mathbf{p}^{\prime}+\left[z^{-1}\right]\right) \tau\left(N-1, n-1, \mathbf{p}^{\prime}-\left[z^{-1}\right]\right) \\
=\frac{1}{2}\left(1-(-1)^{N^{\prime}+N}\right) \tau\left(N^{\prime}, n, \mathbf{p}^{\prime}\right) \tau(N, n, \mathbf{p})
\end{array}
$$

Here $\mathbf{p}=\left(p_{1}, p_{2}, \ldots\right), \mathbf{p}^{\prime}=\left(p_{1}^{\prime}, p_{2}^{\prime}, \ldots\right)$. The notation $\mathbf{p}+\left[z^{-1}\right]$ denotes the set $\left(p_{1}+z^{-1}, p_{2}+z^{-2}, p_{3}+z^{-3}, \ldots\right)$ and $V$ is defined by (11).

Equations (117) are the same as in 32 while equations (116) relate tau functions with different discrete time $n$ and were written down in [58, and 39 .

Taking $N^{\prime}=N+1$ and all $p_{i}=p_{i}^{\prime}, i \neq 2$ in (117) and picking up the terms linear in $p_{2}^{\prime}-p_{2}$ we obtain (66). Taking $N^{\prime}=N+1$ and all $p_{i}=p_{i}^{\prime}, i \neq 1$ in (116) and picking up the terms linear in $p_{1}^{\prime}-p_{1}$ we obtain (67)

The relation of the BKP hierarchy to the two- and three-component KP hierarchy was established in 39 .

\section{B Hypergeometric BKP tau function. Fermionic formulae}

Details may be found in [55,58. Let $\left\{\psi_{i}, \psi_{i}^{\dagger}, i \in \mathbb{Z}\right\}$ are Fermi creation and annihilation operators that satisfy the usual anticommutation relations and vacuum annihilation conditions

$$
\left[\psi_{i}, \psi_{j}\right]_{+}=\delta_{i, j}, \quad \psi_{i}|n\rangle=\psi_{-i-1}|n\rangle=0, \quad i<n
$$

In contrast to the DKP hierarchy introduced in 31 for the BKP hierarchy introduced in 32 one needs an additional Fermi mode $\phi$ which anticommutes with each other Fermi operator except itself: $\phi^{2}=\frac{1}{2}$, 
and $\phi|0\rangle=\frac{1}{\sqrt{2}}|0\rangle$, see [32]. Then the hypergeometric BKP tau function introduced in [58] may be written as

$$
\begin{gathered}
g(n) \tau_{r}^{\mathrm{B}}(N, n, \mathbf{p})=\left\langle n\left|e^{\sum_{m>0} \frac{1}{m} J_{m} p_{m}} e^{\sum_{i<0} U_{i} \psi_{i}^{\dagger} \psi_{i}-\sum_{i \geq 0} U_{i} \psi_{i} \psi_{i}^{\dagger}} e^{\sum_{i>j} \psi_{i} \psi_{j}-\sqrt{2} \phi \sum_{i \in \mathbb{Z}} \psi_{i}}\right| n-N\right\rangle= \\
=\sum_{\substack{\lambda \\
\ell(\lambda) \leq N}} e^{-U_{\lambda}(n)} s_{\lambda}(\mathbf{p})=g(n) \sum_{\substack{\lambda \\
\ell(\lambda) \leq N}} r_{\lambda}(n) s_{\lambda}(\mathbf{p})
\end{gathered}
$$

where $J_{m}=\sum_{i \in \mathbb{Z}} \psi_{i} \psi_{i+m}^{\dagger}, m>0, U_{\lambda}(n)=\sum_{i} U_{h_{i}+n}, r(i)=e^{U_{i-1}-U_{i}}$ and

$$
\begin{aligned}
e^{-U_{0}+\cdots-U_{n-1}} & \text { if } n>0 \\
1 & \text { if } n=0 \\
e^{U_{-1}+\cdots U_{n}} & \text { if } n<0
\end{aligned}
$$$$
g(n):=\left\langle n\left|e^{\sum_{i<0} U_{i} \psi_{i}^{\dagger} \psi_{i}-\sum_{i \geq 0} U_{i} \psi_{i} \psi_{i}^{\dagger}}\right| n\right\rangle=
$$

In (118) the summation runs over all partitions whose length do not exceed $N$.

Remark 23. Let us note that without the additional Fermi mode $\phi$ the summation range in (118) does include partitions with odd partition lengths. One can avoid this restriction by introducing a pair of DKP tau functions which seems unnatural.

Apart of (118) the same series without the restriction $\ell(\lambda) \leq N$ is the example of the BKP tau function however it is related to the single value $n=0$, the $n$-dependence destroys the simple form of such tau function, see [58. 